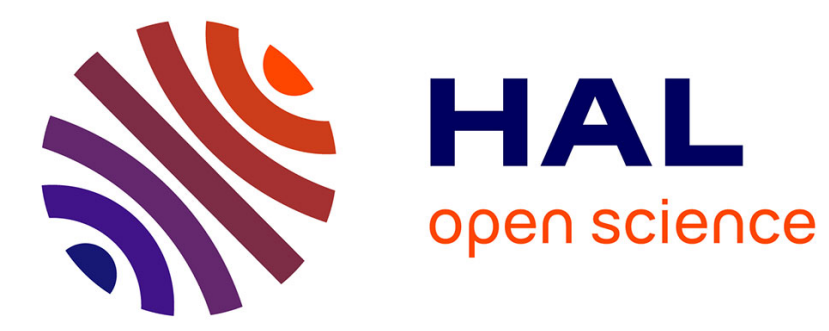

\title{
Disrupting quorum sensing alters social interactions in Chromobacterium violaceum
}

\author{
Sonia Mion, Nathan Carriot, Julien Lopez, Laure Plener, Annick \\ Ortalo-Magné, Eric Chabrière, Gérald Culioli, David Daudé
}

\section{To cite this version:}

Sonia Mion, Nathan Carriot, Julien Lopez, Laure Plener, Annick Ortalo-Magné, et al.. Disrupting quorum sensing alters social interactions in Chromobacterium violaceum. npj Biofilms and Microbiomes, 2021, 7 (1), pp.40. 10.1038/s41522-021-00211-w . hal-03304164

\section{HAL Id: hal-03304164 \\ https://hal-amu.archives-ouvertes.fr/hal-03304164}

Submitted on 6 Sep 2021

HAL is a multi-disciplinary open access archive for the deposit and dissemination of scientific research documents, whether they are published or not. The documents may come from teaching and research institutions in France or abroad, or from public or private research centers.
L'archive ouverte pluridisciplinaire HAL, est destinée au dépôt et à la diffusion de documents scientifiques de niveau recherche, publiés ou non, émanant des établissements d'enseignement et de recherche français ou étrangers, des laboratoires publics ou privés.

\section{(c)(1)}

Distributed under a Creative Commons Attribution| 4.0 International License 
ARTICLE OPEN

Disrupting quorum sensing alters social interactions in Chromobacterium violaceum

\author{
Sonia Mion ${ }^{1,5}$, Nathan Carriot ${ }^{2,5}$, Julien Lopez ${ }^{2}$, Laure Plener ${ }^{3}$, Annick Ortalo-Magné ${ }^{2}$, Eric Chabrière $\mathbb{I}^{1 凶}$, \\ Gérald Culioli $\mathbb{1}^{2,4 凶}$ and David Daudé $\mathbb{1}^{3 凶}$
}

Quorum sensing (QS) is a communication system used by bacteria to coordinate a wide panel of biological functions in a cell density-dependent manner. The Gram-negative Chromobacterium violaceum has previously been shown to use an acyl-homoserine lactone (AHL)-based QS to regulate various behaviors, including the production of proteases, hydrogen cyanide, or antimicrobial compounds such as violacein. By using combined metabolomic and proteomic approaches, we demonstrated that QS modulates the production of antimicrobial and toxic compounds in C. violaceum ATCC 12472. We provided the first evidence of anisomycin antibiotic production by this strain as well as evidence of its regulation by QS and identified new AHLs produced by C. violaceum ATCC 12472. Furthermore, we demonstrated that targeting AHLs with lactonase leads to major QS disruption yielding significant molecular and phenotypic changes. These modifications resulted in drastic changes in social interactions between $C$. violaceum and a Gram-positive bacterium (Bacillus cereus), a yeast (Saccharomyces cerevisiae), immune cells (murine macrophages), and an animal model (planarian Schmidtea mediterranea). These results underscored that AHL-based QS plays a key role in the capacity of $C$. violaceum to interact with micro- and macroorganisms and that quorum quenching can affect microbial population dynamics beyond AHL-producing bacteria and Gram-negative bacteria.

npj Biofilms and Microbiomes (2021)7:40; https://doi.org/10.1038/s41522-021-00211-w

\section{INTRODUCTION}

Many proteobacteria use acyl-homoserine lactones (AHLs) to orchestrate their behavior in a cell density-dependent manner ${ }^{1}$. This communication system, referred to as quorum sensing (QS), plays a key role in bacterial adaptation to the environment. QS is involved in many mechanisms, including biofilm formation, production of virulence factors, and biosynthesis of antimicrobial compounds, such as phenazine ${ }^{2}$, bactobolin ${ }^{3}$, or violacein ${ }^{4}$. Furthermore, several studies have shown that QS plays a part not only in intra- or interspecies bacterial interactions ${ }^{1,5,6}$ but also in interkingdom interactions ${ }^{7}$.

Disrupting QS has appeared as a promising way to counteract bacterial behavior in fields of application useful for human healthcare and agriculture ${ }^{8}$. To this end, many strategies have been considered, including inhibition of QS signals using chemical inhibitors (QSI), their sequestration by antibodies, or the use of quorum-quenching enzymes (QQE). QQE have been particularly investigated as they can be used extracellularly to interact with signaling molecules, especially AHLs. Among QQE, lactonases, and especially those issued from extreme environments, have been shown to efficiently alter social behaviors in many proteobacteria, including Pseudomonas aeruginosa $a^{9,10}$, Acinetobacter baumannii $^{11,12}$, Burkholderia cenocepacia ${ }^{13}$, and Chromobacterium violaceum $^{14}$. Although most studies involving lactonases relied on the use of pure bacterial cultures, recent reports underlined that these biocatalysts may impact complex bacterial communities both in vitro and in situ ${ }^{15,16}$. Lactonase-mediated QS disruption was shown to change bacterial population dynamics and concomitantly to reduce biofilm formation in a recirculating bioreactor ${ }^{15}$ and also to reduce biocorrosion induced by microbial communities on steel surfaces immersed in a lake water ${ }^{16}$. Strikingly, metagenomic analyses revealed that lactonases did not only affect the proportion of AHL-using proteobacteria but also Gram-positive and -negative bacteria that are not known to use or sense AHLs. These results suggest that altering AHL-based QS with lactonases may drive global population changes although the mechanisms and molecular determinants of these alterations remain poorly understood.

Considering the abundance of QS-regulated traits, particularly those involved in the biosynthesis of antimicrobials, the use of exogenous QQE appeared to be a relevant approach to modulate bacterial interspecies competition ${ }^{17}$.

Here, we considered the use of the lactonase SsoPox W263I, a QQE able to degrade a wide spectrum of AHLs and to affect microbial communities from soil and freshwater ecosystems, to decipher how QQ can modulate molecular mechanisms involved in social interactions ${ }^{18}$ in a single proteobacteria species representative from these ecosystems. The impact of the lactonase SsoPox W263I on the model environmental bacterium C. violaceum ATCC 12472 (CV 12472) ${ }^{19,20}$ was thus investigated. Through a multi-omics approach, coupling metabolomics and proteomics together with phenotypic and microbiological investigations, the impact of enzymatic quenching on the strain behavior was deeply analyzed with a special focus on the production of antibiotic compounds ${ }^{21}$. We reported the production of newly observed AHLs and described, for the first time, the production of the antibiotic anisomycin in CV 12472. We further provided a series of experiments suggesting that SsoPox W263I alters the ability of C. violaceum to produce QS-regulated antimicrobials and to compete with prokaryotic and eukaryotic

\footnotetext{
${ }^{1}$ Aix Marseille University, Institut de Recherche pour le Développement, Assistance Publique - Hôpitaux de Marseille, Microbes Evolution Phylogeny and Infections, Institut Hospitalo-Universitaire-Méditerranée Infection, Marseille, France. ${ }^{2}$ Université de Toulon, MAPIEM, Toulon, France. ${ }^{3}$ Gene\&GreenTK, Marseille, France. ${ }^{4}$ Institut Méditerranéen de Biodiversité et d'Ecologie marine et continentale, UMR CNRS-IRD, Avignon Université, Aix-Marseille Université, Avignon, France. ${ }^{5}$ These authors contributed equally: Sonia Mion, Nathan Carriot. ${ }^{凶}$ email: eric.chabriere@univ-amu.fr; gerald.culioli@univ-avignon.fr; david.daude@gene-greentk.com
} 

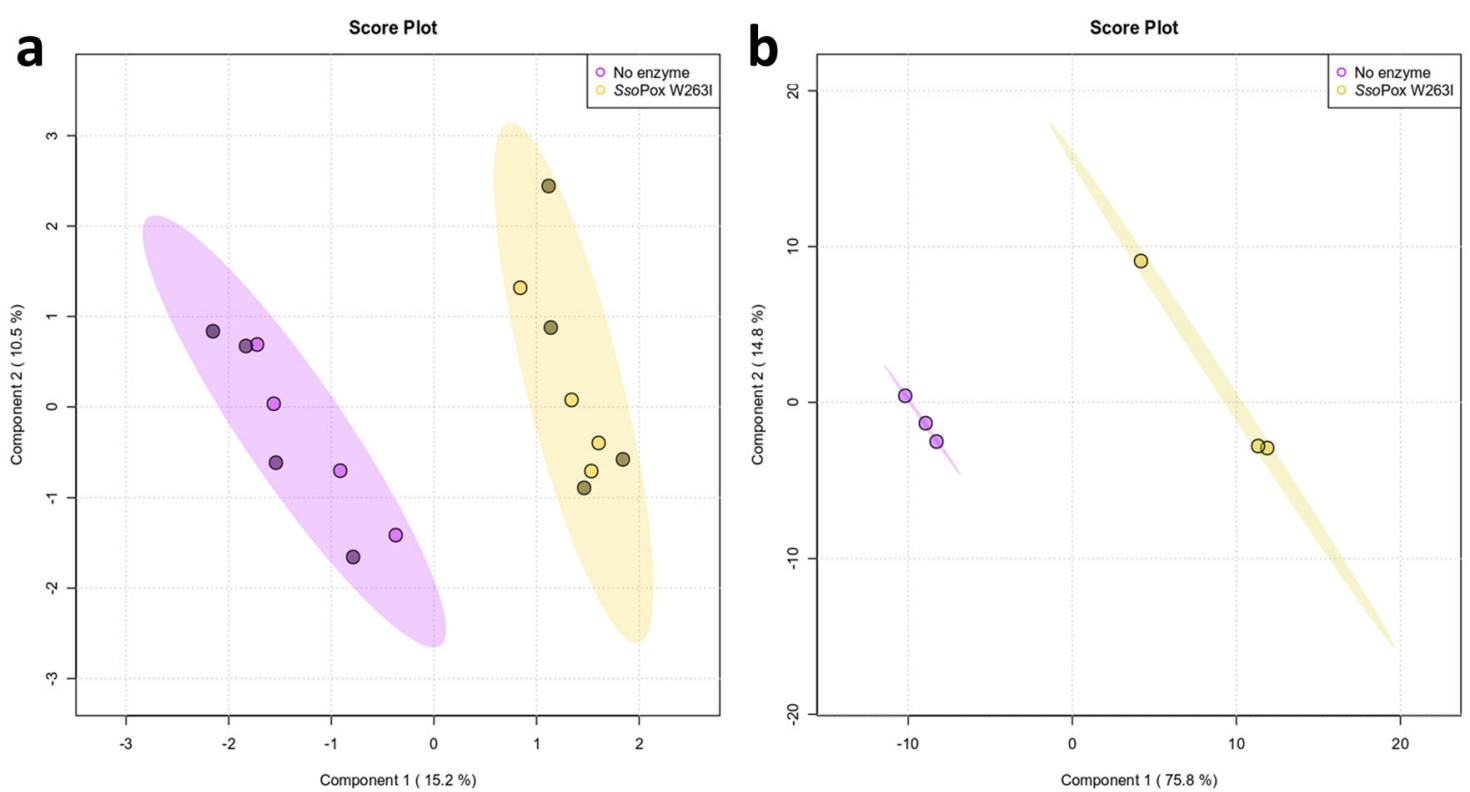

Fig. 1 PLS-DA analysis of proteomic and metabolomic data of $C$. violaceum cultures with and without lactonase treatment. Score plots of PLS-DA analysis of proteomic (a) $(n=8$ : four biological replicates $\times$ two technical replicates indicated by darker point) and metabolomic data (b) ( $n=3$ : three biological replicates) of SsoPox W263l $\left(0.5 \mathrm{mg} \mathrm{ml}^{-1}\right)$ treated samples (yellow) and untreated ones as negative controls (purple).

organisms. Taken as a whole, these results indicate that disrupting QS may broadly impact interspecies interactions and microbial population dynamics.

\section{RESULTS}

\section{Lactonase SsoPox W263I modulates C. violaceum proteome}

Proteomes of CV 12472 with and without treatment by the QQ lactonase SsoPox W263I were analyzed. The use of a statistical PLSDA analysis revealed a significant impact of the enzyme on the proteome of CV 12472 as the control and treated groups were found distinctly separated on the first component $(15.2 \%$ of the total variance) (Fig. 1a).

A total of 889 out of the 4397 (i.e., 20.2\%) proteins of CV 12472 were detected and identified (Uniprot database) (Supplementary Data 1). These 889 proteins were clustered by biological functions according to the GO database using the DAVID bioinformatic tool $^{22}$ (Fig. 2a). This method allowed to assign of biological functions to $82.6 \%$ of this set of proteins. Proteins were mainly involved in metabolic processes (31\%), followed by translation (8.3\%) and transcription (6.6\%).

Relative abundances of 38 proteins were found to be significantly impacted upon SsoPox W263I treatment (i.e., fold change $\geq 1.5$ and $P$ value $<0.05$ ) (Fig. $2 b, c$ ) and were further clustered by biological function (Fig. 2b). Among these proteins, ten were associated with metabolic pathways, including amino acids and cofactor biosynthesis as well as the metabolism of carbohydrates, energy, and fatty acids. One protein was detected in each of the following functions: translation mechanisms, DNA repair and replication, cell cycle, and protein modification and degradation. The abundance of five proteins involved in membrane transport and secretion systems decreased upon enzymatic treatment. Interestingly, changes in proteins involved in the biosynthesis of diverse cytotoxic compounds such as hydrogen cyanide, violacein, and anisomycin were observed. These latter proteins were all downregulated by SsoPox W263I treatment (Fig. 2c). Finally, twelve proteins significantly affected by the enzymatic treatment remained unknown or uncharacterized.

\section{SsoPox W263I changes the metabolomic profile of $C$. violaceum}

To further associate proteome modifications to molecular changes, metabolomic investigations were undertaken. LC-(+)-ESI-MS metabolomic analysis enabled the identification of a primary dataset of $8496 \mathrm{~m} / \mathrm{z}$ features from raw LC-MS data, which was further refined and reduced down to a final dataset of 406 metabolites thanks to three filtration steps (Supplementary Table 1). The concentration of several metabolites was found to be significantly different between enzymatically treated and control samples (Fig. 3a).

PLS-DA analysis revealed two distinct groups formed by treated and control samples, separated on the first component $(75.8 \%$ of the total variance) (Fig. 1b), thus indicating a significant effect of the enzymatic treatment on the metabolome of CV 12472. The PLS-DA model showed high predictive accuracy and relevance with $R^{2}=0.94$ and $Q^{2}=0.84$. This analysis was used to determine the variable importance in projection (VIP) score values, indicating the importance of each $\mathrm{m} / \mathrm{z}$ feature in the discrimination between treated and control samples. Twenty-one $\mathrm{m} / \mathrm{z}$ features with the higher VIP scores (VIP score >1.5) were selected (Fig. 3a), and the corresponding molecular formulae were determined based on accurate mass measurement, isotopic patterns, and MS/MS data analysis. In most cases, a putative identification was proposed (Supplementary Table 2).

Global Natural Products Social (GNPS) molecular networking workflow was further used to organize the whole MS/MS metabolomic dataset so as to obtain a global view of the different groups of chemical compounds (called "clusters") containing VIPs (Fig. $3 \mathrm{~b})^{23}$ for annotation purposes. Such a representation allowed to gather metabolites with similar MS/MS data, and thus with putatively close chemical structures, in the same cluster. Cosine score (CS) and minimum matched fragment ions (MF) are crucial parameters to link similar metabolites together (called "nodes") and then to build molecular networks. In this study, the use of low values of CS and MF (CS $=0.7, M F=$ 4) was adopted to create a maximum of links between annotated nodes and non-identified VIP nodes ${ }^{24}$. Some nodes observed into AHLs-, violacein-, and anisomycin-containing clusters were then identified and will be further discussed. Through this 
a

C

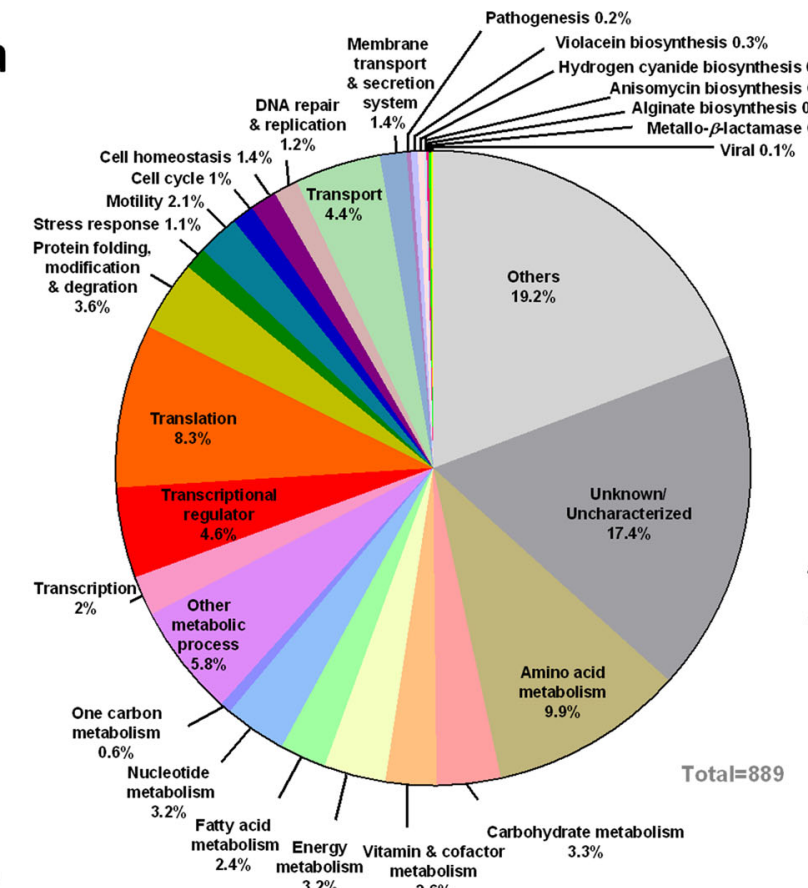

b

$0.3 \%$ :

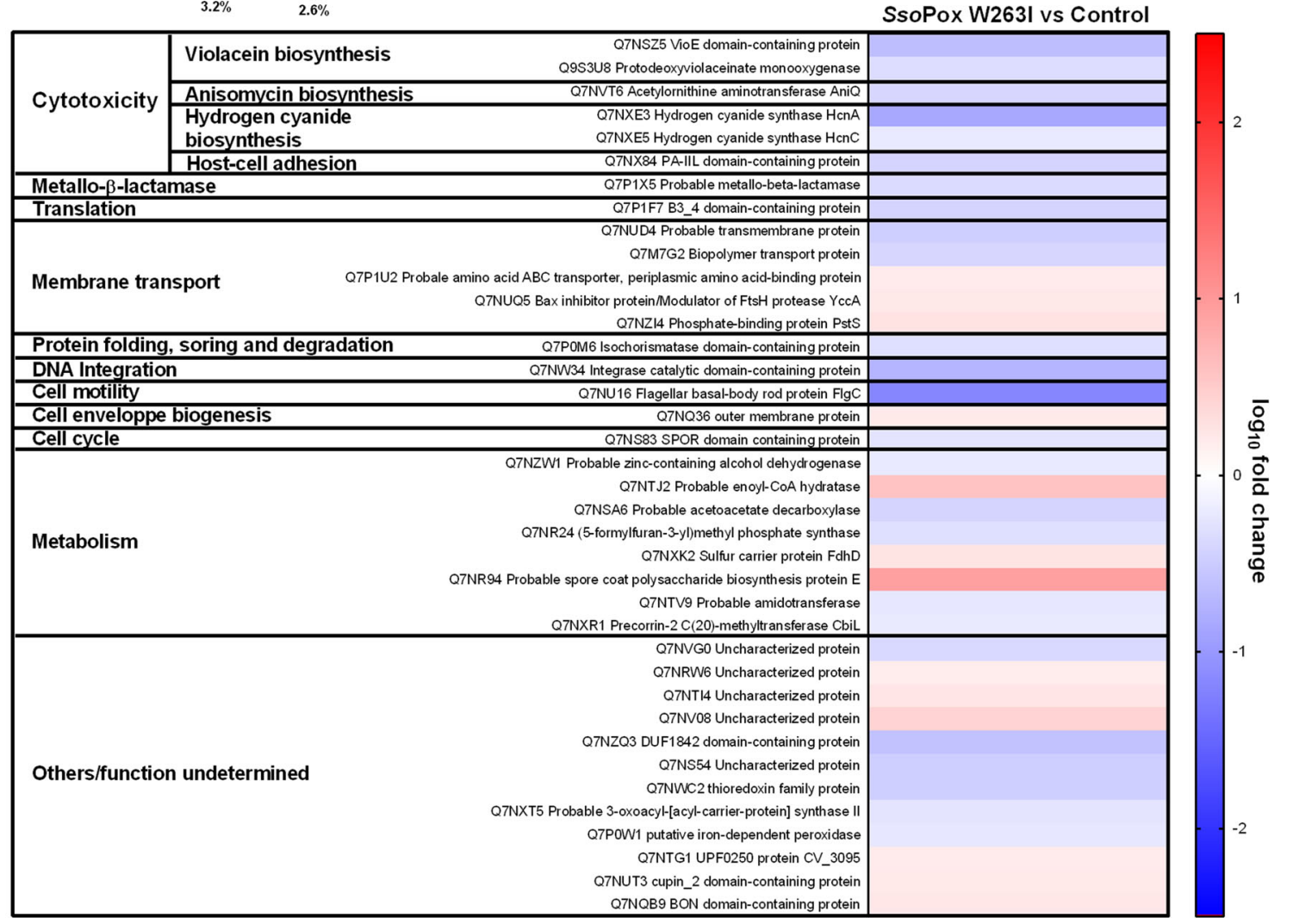

Fig. 2 Proteome analysis of $\boldsymbol{C}$. violaceum cultures with and without lactonase treatment. a Functional distribution of all proteins detected in C. violaceum samples. b Functional distribution of proteins significantly impacted by SsoPox W263I treatment. c Impact of SsoPox W263I treatment on C. violaceum protein production. Heatmap represents the $\log _{10}$ fold change in protein production of SsoPox W263I-treated samples as compared to untreated ones as negative controls (selection criteria for significantly impacted proteins were $P$ value $<0.05$ and fold change $\geq 1.5$ ). Down- and upregulated protein expressions are presented in blue and red, respectively.

analytical procedure, more than sixty metabolites of the metabolome of CV 12472 were identified, some of which being among the VIPs that will be important in the following discussions.

\section{SsoPox W263I hydrolyzes AHLs produced by C. violaceum}

Both metabolome and proteome analyses revealed a significant impact of SsoPox W263I on CV 12472 metabolism. To evaluate whether these discrepancies are related to QS disruption, AHLs 


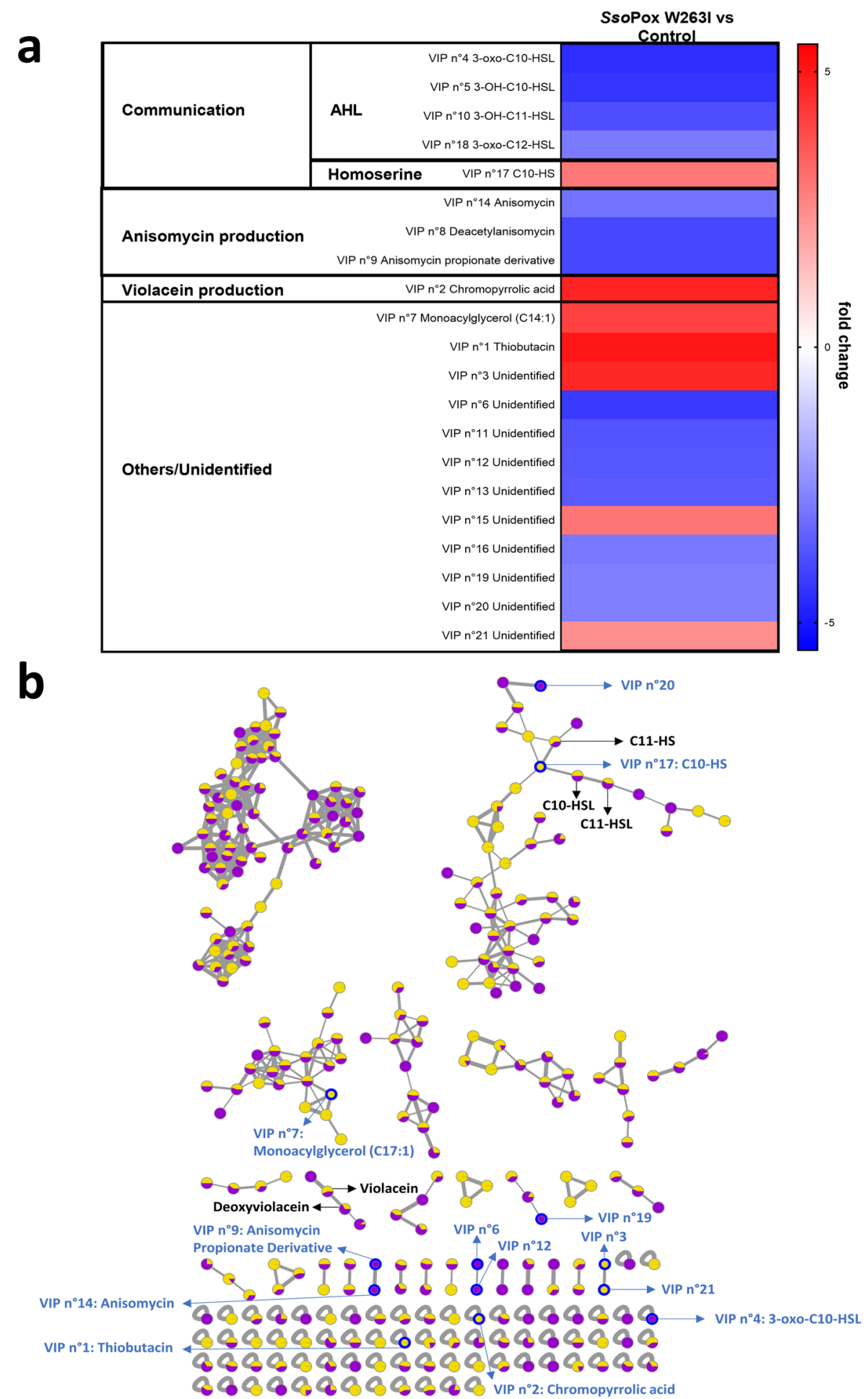

Fig. 3 Metabolome analysis of $C$. violaceum cultures with and without lactonase treatment. a Impact of SsoPox W263I treatment on $C$. violaceum metabolite production. Heatmap represents the fold change of the 21 most discriminating metabolites (identified by their VIP score through the PLS-DA analysis) between SsoPox W263l $\left(0.5 \mathrm{mg} \mathrm{m}^{-1}\right)$ treated samples and untreated ones as negative controls. Down- and upregulated metabolite expressions are presented in blue and red, respectively. b Molecular network of LC-MS metabolomic data and identification of molecules of interest in clusters. VIPs are circled in blue. For each metabolite, color ratio corresponds to its relative abundance in treated (yellow) and untreated (purple) samples.

and their potential degradation products were specifically analyzed in the cultures of CV 12472.

This analysis of whole-culture extracts led to the identification of several AHLs (Fig. 4). Among these, the main AHLs observed on the chromatograms, and already known to be produced by CV 12472 , were $\mathrm{N}$-decanoyl-homoserine lactone (C10-HSL) and $\mathrm{N}$-(3hydroxydecanoyl)-homoserine lactone (3-OH-C10-HSL) ${ }^{19-21,25}$. Furthermore, non-targeted metabolomics allowed the detection 
a

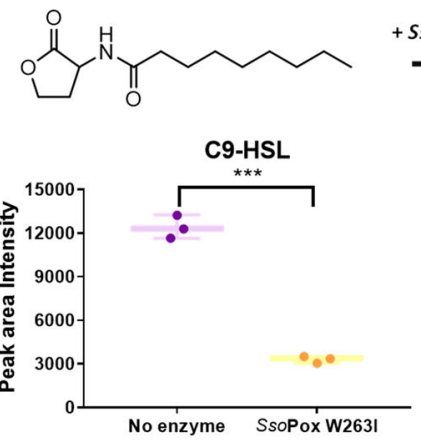

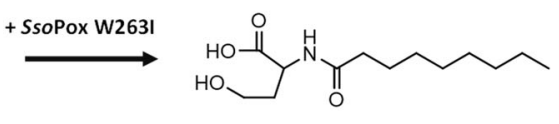

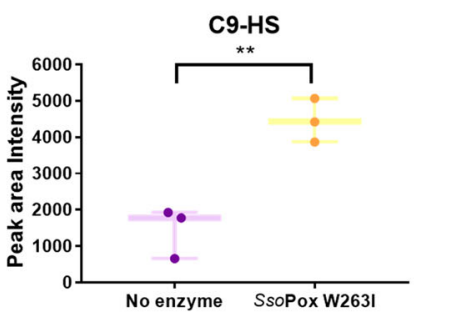

No enzyme SsoPox W263I

b

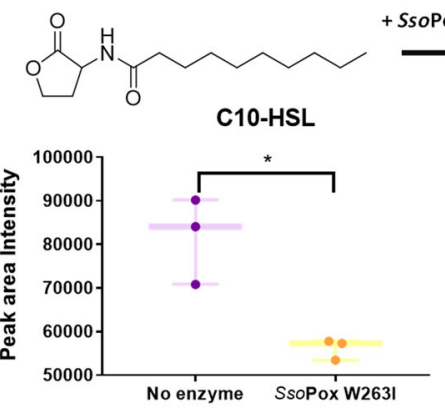

C

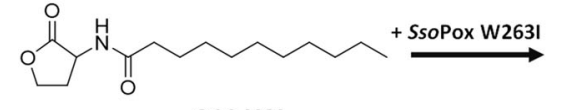

C11-HSL

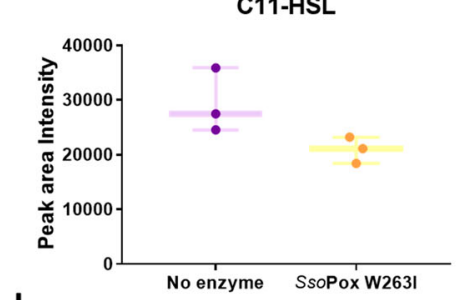

d

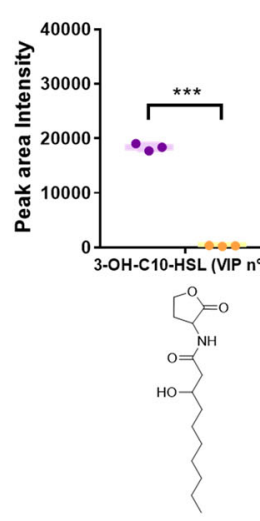

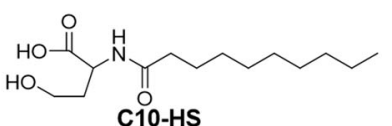

C10-HS

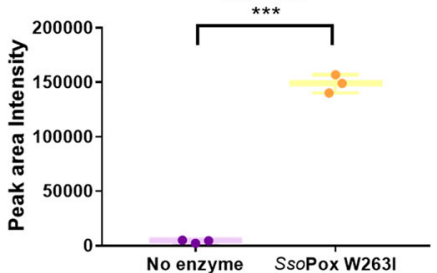

HOC

C11-HS
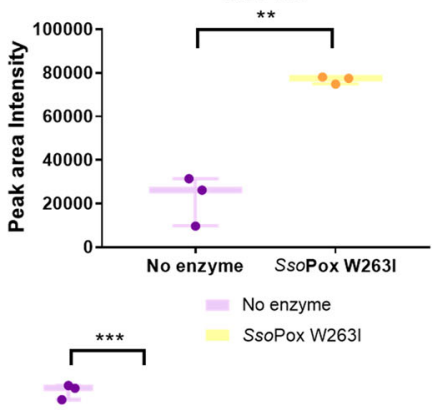

SsoPox W263!

Fig. 4 SsoPox W263I impacts production of AHLs in CV 12472. Chemical structures and LC-MS peak area intensities of AHLs and their hydrolyzed form in control (purple) and treated samples (yellow). a C9-HSL, b C10-HSL, c C11-HSL, and d other AHLs. Bars represent the mean and standard deviations of peak area intensities on the corresponding LC-MS chromatograms in three biological replicates (each represented by one point). ${ }^{*} P$ value $<0.05,{ }^{* *} P$ value $<0.01,{ }^{* *} P$ value $<0.001$ according to Student's $t$ test.

and identification of AHLs reported to our knowledge, for the first time in CV 12472: $N$-nonanoyl-homoserine lactone (C9-HSL), $\mathrm{N}$-undecanoyl-homoserine lactone (C11-HSL), N-(3-oxodecanoyl)homoserine lactone (3-oxo-C10-HSL), N-(3-hydroxyundecanoyl)homoserine lactone (3-OH-C11-HSL), and $\mathrm{N}$-(3-oxododecanoyl)homoserine lactone (3-oxo-C12-HSL). In addition, while $\mathrm{N}$-dodecanoyl-homoserine lactone (C12-HSL) was not observed, its open form $\mathrm{N}$-dodecanoyl-homoserine (C12-HS) was detected. Supernatant extracts obtained after removal of bacterial cells by centrifugation were also analyzed by LC-MS and allowed the detection of the same AHLs in the same proportions as those observed in the whole-culture extracts (Supplementary Fig. 1a).

The detection of diverse AHLs in CV 12472, albeit reported for the first time, is comparable to many bacterial species known to 
use different $A H L s$ to regulate their $\mathrm{QS}^{26}$. Although $\mathrm{C} 10-\mathrm{HSL}$ and 3$\mathrm{OH}-\mathrm{C} 10-\mathrm{HSL}$ have been previously reported to be the main AHLs produced by CV 12472, other AHLs including 3-oxo-C10-HSL and 3-oxo-C12-HSL, have been shown to exogenously increase the production of violacein in CV $12472^{19}$. C11-HSL and 3-OH-C11-HSL are not common in soil bacteria and have mainly been reported in Vibrionaceae species ${ }^{27,28}$. C9-HSL is also rarely detected in bacteria but has already been reported in Burkholderia cepacia strain GG4 isolated from the ginger rhizosphere ${ }^{29}$, and in Vibrio fischeri (also called Aliivibrio fischeri) strains associated with the squid Euprymna scolope $^{30}$. Interestingly, C6-HSL, a molecule described as the cognate AHL used by ATCC $31532^{20}$, another well-described strain of $C$. violaceum, was not detected in this study, adding proof that the array of AHLs generated by $C$. violaceum appears to be strainspecific.

Although $\mathrm{C} 10-\mathrm{HSL}$, and to a lesser extent 3-OH-C10-HSL and 3oxo-C10-HSL, appeared as the main AHLs of CV 12472 wholeculture and supernatant extracts (Supplementary Fig. 1a), respective quantities of each detected AHLs and their specific impact on CV 12472 behavior are not precisely known. Further studies are necessary to determine each $\mathrm{AHL}$ exact concentration and contribution to QS or if some may represent inactive metabolic intermediates.

This study focused on the impact of QQE on CV 12472 behavior, to do so the impact of SsoPox W263I on the relative abundance of AHLs was further analyzed. The quantity of all detected AHLs decreased significantly in the treated samples (Fig. 4). Furthermore, the open forms of C9-HSL, C10-HSL, C11-HSL, and C12-HSL, respectively, $\mathrm{N}$-nonanoyl-homoserine (C9-HS), $\mathrm{N}$-decanoyl-homoserine (C10-HS), N-undecanoyl-homoserine (C11-HS), and C12-HS, were detected in greater amounts in treated samples than in untreated ones (Fig. $4 a-c$ ). Such an observation is consistent with the previously reported mechanism of SsoPox ${ }^{31}$ and with the catalytic performance of SsoPox W263I toward the AHLs detected in this study (Supplementary Table 3) and the autoinduction mechanism described in CV $12472^{21}$.

These results confirmed the hydrolytic action of SsoPox W263I on the AHLs produced by CV 12472 and highlighted its QQ potential.

\section{Lactonase drastically decreases the production of known QS- associated factors}

To evaluate how molecular modifications could be related to phenotypic variations, the impact of SsoPox W263I was investigated on QS-related behaviors of CV 12472 (Fig. 5).

C. violaceum is known to produce the characteristic purple pigment violacein under the regulation of $\mathrm{QS}^{19,20}$. As expected, the addition of a lactonase to the culture medium suppresses the production of this pigment (Fig. 5a). Linking metabolomic and proteomic analyses allowed us to observe the impact of enzymatic treatment on different steps of the biosynthetic pathway of violacein (Fig. 6 and Supplementary Fig. 2). The main benefits of the QQ treatment were a fourfold decrease in VioE production and a 700-fold increase of chromopyrrolic acid. This latter is known to be formed spontaneously in the absence of VioE (Fig. 6) ${ }^{32,33}$ and was thus found to be significantly negatively correlated to this protein (Fig. 5a). Together with VioE, the abundance of VioD was significantly reduced, leading to a drastic decrease in the production of violacein and its derivatives (i.e., deoxyviolacein, proviolacein, prodeoxyviolacein, and deoxyviolacein) (Fig. 6 and Supplementary Fig. 2). Proteomic and metabolomic data matrices were further processed by regularized Canonical Correlation Analysis (rCCA) $)^{34-36}$ with a cut-off value of 0.5 chosen to create a multi-omics correlation network. This network showed significant positive relationships between the proteins VioD and VioE, on the one hand, and violacein, deoxyviolacein, and the AHLs, on the other, while these two proteins were negatively correlated with chromopyrrolic acid and the hydrolyzed AHLs (Fig. 5a).

Chromobacterium species are also known for their ability to produce the toxic metabolite hydrogen cyanide ${ }^{37,38}$. This production is under QS regulation in C. violaceum ${ }^{21}$. In this study, enzymatic treatment reduced by $>50 \%$ the level of hydrogen cyanide in the supernatant (Fig. $5 \mathrm{~b}$ ). $\mathrm{HcnC}$ and $\mathrm{HcnA}$, two proteins involved in hydrogen cyanide biosynthesis ${ }^{39}$, were found significantly downregulated by SsoPox W263I treatment (fold change of -7.27 and -1.65 , respectively, as compared to control) (Fig. 2c) and their variations were positively correlated with AHLs and negatively correlated with hydrolyzed AHLs (Fig. 5b).

Finally, a strong inhibitory effect of the lactonase on the production of proteases as well as on the biofilm formation was also observed with fold changes of -6 and -17 , respectively. Nevertheless, neither proteins nor metabolites could be clearly associated with these phenotypes (Fig. 5c, d). Altogether, these results suggested that SsoPox W263I constitutes a potent QQ agent for the bacterial strain CV 12472, inducing major phenotypic and metabolic changes.

\section{Combined omics analysis identifies anisomycin as a new QS- regulated factor}

Besides known QS-regulated metabolites of CV 12472, further analysis of the metabolome of CV 12472 led to the identification of anisomycin, deacetylanisomycin, and anisomycin propionate derivative molecules (Figs. 3 and 7). Anisomycin is an antibiotic interfering with proteins and DNA synthesis in eukaryotes and is mainly described in Streptomyces species ${ }^{40}$. Recently, the biosynthetic gene cluster of anisomycin in Streptomyces hygrospinosus var. beijingensis has been reported. It mainly consists in five proteins (AniQ, AniP, AniN, Anil, and AniK) for the biosynthesis of anisomycin and two additional proteins being involved in its glycosylation. Homologs of AniQ, AniP, and AniN have been previously identified in C. violaceum genome but the production of anisomycin by $C$. violaceum and the functionality of the identified homologs have not been confirmed ${ }^{41}$. Here, we provided the first evidence of anisomycin production in CV 12472 and a thorough comparison of S. hygrospinosus ACCC40033 and CV 12472 anisomycin gene clusters. Our data confirmed the identification of the biosynthetic gene cluster of anisomycin in the CV 12472 genome between the loci CV 2255 and CV 2262. Anisomycin was also identified in cell-free supernatants (Supplementary Fig. 1b), suggesting that CV 12472 is also equipped for the secretion of this antibiotic. This analysis revealed that $C$. violaceum possesses all the required proteins to biosynthesize anisomycin from L-tyrosine although glycosylation proteins were not identified (Supplementary Fig. 3). Here, we further showed, by combining proteomic and metabolomic analyses, that anisomycin was drastically downregulated by QQ treatment (Fig. 7). Not all molecules involved in anisomycin biosynthesis were detected by the multi-omics approach, but insightful observations highlighted the impact of SsoPox W263I on its production. The protein CV_2256, now identified as AniQ and involved in the formation of several biosynthetic intermediates of anisomycin, was found significantly downregulated by the enzymatic treatment (Fig. 7a). Deacetylanisomycin, a precursor of anisomycin was also downregulated, along with the anisomycin propionate derivative (also known as 3097-B1, formed by an unknown mechanism and already detected in Streptomyces strain SA309742) (Fig. 7a). AniQ, deacetylanisomycin, anisomycin and its propionate derivative, were all positively correlated with the production of AHLs and negatively correlated with the occurrence of hydrolyzed AHLs (Fig. 7b).

Altogether, the results of this multi-omics approach showed that CV 12472 can biosynthesize anisomycin and that this production is under QS regulation. 


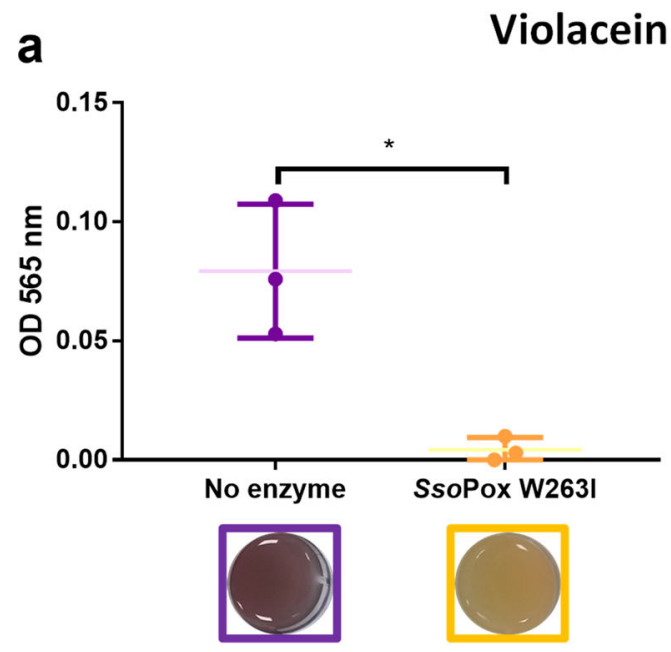

\section{production}
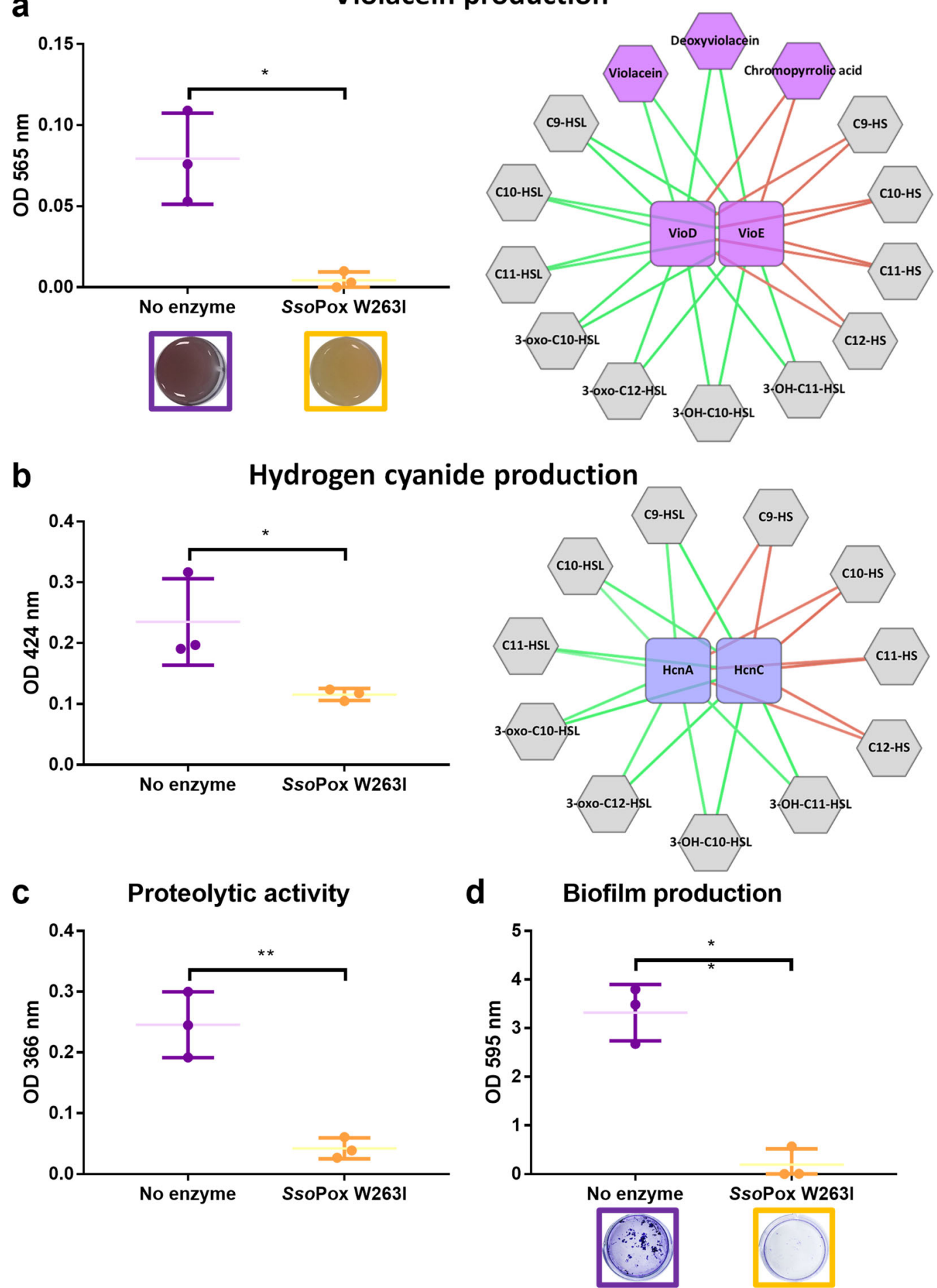

Fig. 5 SsoPox W263I inhibits QS-regulated factors production of C. violaceum. Mean levels of violacein (a) hydrogen cyanide (b), protease (c), and biofilm (d) production of C. violaceum untreated (purple) and treated cultures with $0.5 \mathrm{mg} \mathrm{ml}^{-1}$ SsoPox W263l (yellow). Error bars represent the standard deviations of $n=3$ biological replicates (each represented by one point). ${ }^{*} P$ values $<0.05$; ${ }^{* *} P$ values $<0.01$ according to Student's $t$ test. Representative pictures of untreated (yellow squares) and lactonase treated cultures (yellow squares) (a) and their biofilm formation after coloration with crystal violet (d) are given. Network represents correlation multi-omics networks where proteins (squares) and metabolites (hexagons) involved in violacein (a) and hydrogen cyanide production (b) production and significantly impacted by SsoPox W263I are positively (green) or negatively (red) connected.

\section{C. violaceum toxicity toward Gram-positive Bacillus cereus is inhibited by $\mathbf{Q Q}$}

C. violaceum uses QS to induce antimicrobial metabolites production, including the Gram-positive targeting violacein and hydrogen cyanide ${ }^{43,44}$. To evaluate how $Q Q$ may affect the capacity of CV 12472 to compete with invaders, a co-culture model with Bacillus cereus was developed and tested with and without lactonase. Without enzymatic treatment, CV 12472 was 


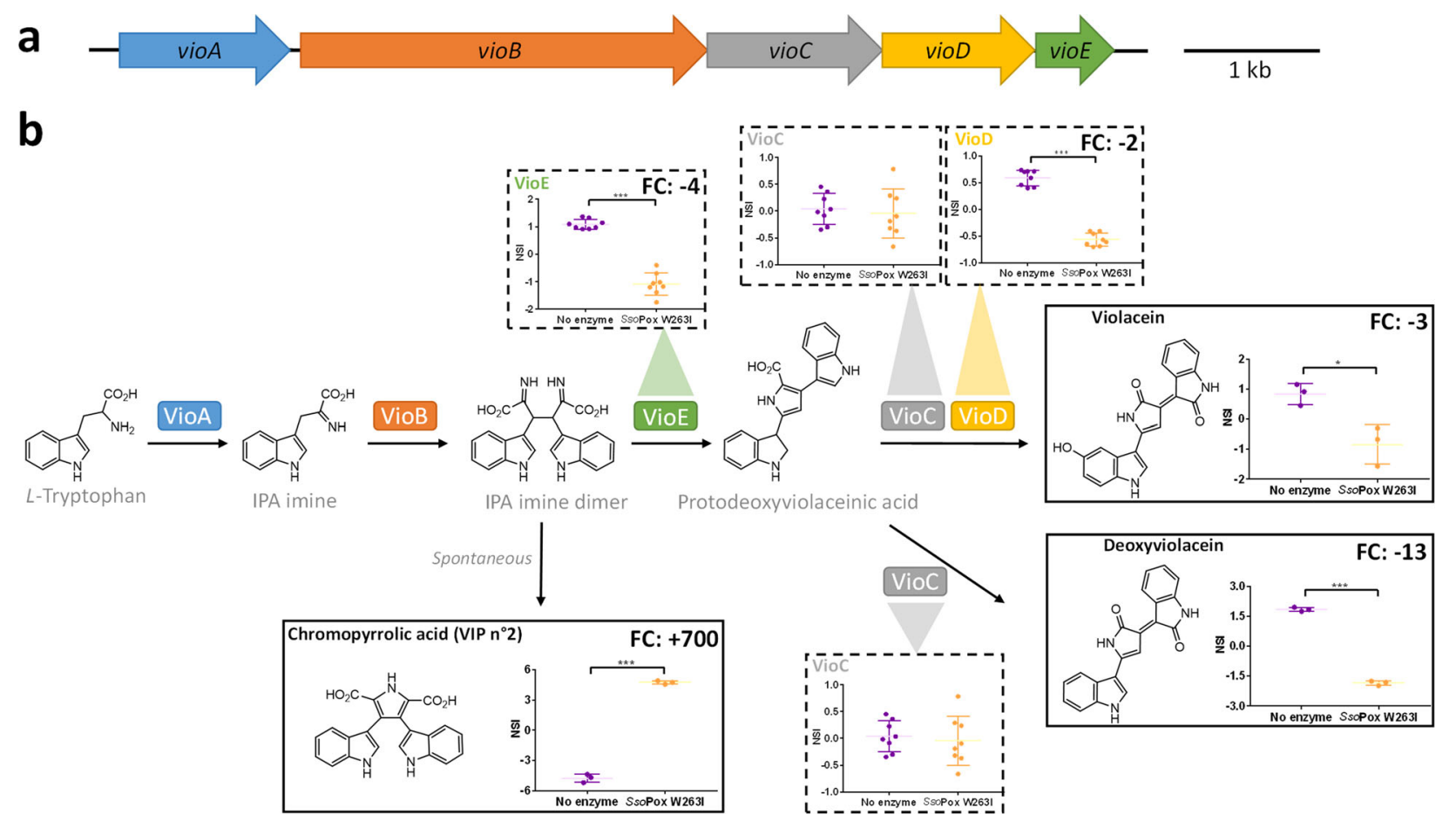

Fig. 6 Impact of SsoPox W263I on violacein biosynthesis in C. violaceum. a Organization of the violacein biosynthesis operon. Arrows indicate the direction of transcription. b Simplified representation of SsoPox W263I impact on violacein biosynthesis by CV 12472 untreated (purple) and treated cultures (yellow). Proteins (dashed line boxes) and metabolites (full-line boxes) normalized scaled intensities (NSI) are represented as the mean and standard deviations (Metabolomic: $n=3$ biological replicates, Proteomic: $n=8,4$ biological replicates $\times 2$ technical replicates). ${ }^{*} P$ value $<0.05,{ }^{* *} P$ value $<0.01,{ }^{* *} P$ value $<0.001$ according to Student's $t$ test. FC fold change.

lethal to $B$. cereus and completely inhibited its growth. Conversely, when CV 12472 was enzymatically quenched, B. cereus was able to grow (Fig. 8a). Similar results were obtained when $B$. cereus was grown in the presence of $50 \%$ CV 12472 supernatant, demonstrating that $C$. violaceum produced one or several extracellular toxic compounds for B. cereus under QS regulation (Supplementary Fig. $4 a)$. These effects could be due to the impact of SsoPox W263I on different QS-regulated mechanisms of CV 12472, such as violacein or hydrogen cyanide production. Violacein is known to disrupt Gram-positive strains' cellular membrane integrity ${ }^{45}$ and hydrogen cyanide is also known to be toxic to some bacterial strains and to be involved in bacterial competition ${ }^{43}$. The downregulation of hydrogen cyanide and violacein production by SsoPox W263I could indeed play a significant role in altering the ability of CV 12472 to eliminate $B$. cereus.

To determine whether these metabolites were involved in the toxicity against $B$. cereus, hydrogen cyanide and violacein were evaluated independently (Supplementary Fig. 4b, c). First, the amounts of violacein and hydrogen cyanide were estimated in CV 12472 supernatants: violacein was found at a concentration of $4 \pm$ $1 \mu \mathrm{g} \mathrm{ml}^{-1}$ in untreated cultures and $0.3 \pm 0.1 \mu \mathrm{g} \mathrm{ml}^{-1}$ in treated ones while hydrogen cyanide concentration was $7 \pm 1 \mu \mathrm{g} \mathrm{ml}^{-1}$ in untreated cultures and $3 \pm 1 \mathrm{\mu g} \mathrm{ml}^{-1}$ in treated ones. The effect of both molecules on $B$. cereus growth was assayed at the concentration found in CV 12472 supernatants. At $4 \mu \mathrm{g} \mathrm{ml}^{-1}$, violacein inhibited $B$. cereus growth in the same proportion as in untreated supernatants. Consistently, as for the treated cultures of CV 12472, a concentration of $0.3 \mu \mathrm{g} \mathrm{ml}^{-1}$ violacein did not affect the growth of $B$. cereus (Supplementary Fig. 4b). Conversely, hydrogen cyanide had no impact on $B$. cereus growth at any tested concentrations (Supplementary Fig. 4c). These results underlined that the toxic effect of CV 12472 supernatants on B. cereus could be mainly attributed to violacein and this effect is drastically decreased by SsoPox W263I.

\section{SsoPox inhibits C. violaceum toxicity toward unicellular eukaryote Saccharomyces cerevisiae}

A competition model was developed using the yeast $S$. cerevisiae to evaluate the potential impact of QS on the interactions of CV 12472 with unicellular eukaryotic organisms (Fig. 8b). The experiment revealed that yeast growth was decreased on YPDAgar inoculated with CV 12472. Treatment of CV 12472 with lactonase inhibited the toxicity of the bacterium toward the yeast and growth increased by a $2.2 \log _{10}$-fold change to reach a similar level as growth on uninoculated YPD-Agar. These results showed that QS regulates the antifungal effect of CV 12472 and can be affected by AHL-targeting QQ. Different antifungal mechanisms could be involved such as violacein ${ }^{46}$ and cyanide production ${ }^{47}$; both under QS regulation in CV 12472 and inhibited by SsoPox W263I. Anisomycin is also an antifungal metabolite, with known toxic activity against S. cerevisiae ${ }^{41}$. Its effect on yeast was tested to evaluate whether it could be responsible for the inhibition of growth caused by CV 12472. Interestingly, we observed that anisomycin induced the formation of small-sized colonies of $S$. cerevisiae while the number of colonies was not affected (Supplementary Fig. 5). Such a morphological change was also observed in our $C$. violaceum vs $S$. cerevisiae competition assay. Small-colony variants have been previously reported in $S$. cerevisiae treated with anisomycin ${ }^{48}$. Therefore, the small-colony variants observed in S. cerevisiae in presence of CV 12472 might be imputable to anisomycin production, but cannot explain the yeast growth defect indicating that, besides anisomycin, at least another toxic compound might be involved in the competition between CV 12472 and S. cerevisiae. Interestingly, cell-free supernatants obtained from treated and untreated cultures of CV 12472 induced the formation of yeast small colonies but did not alter yeast growth, suggesting that growth inhibition might require direct contact between these microorganisms (Supplementary Fig. 5). 


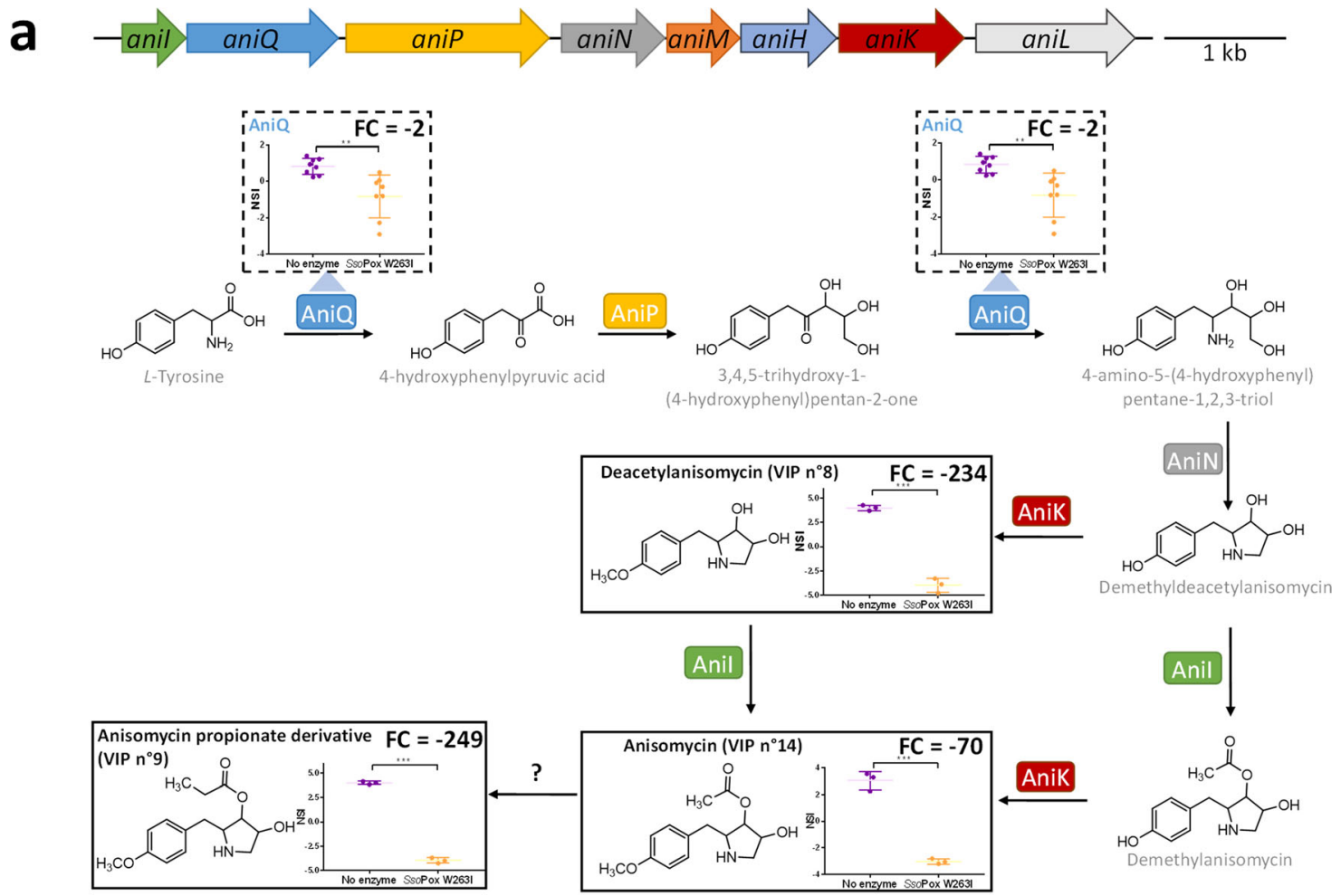

b

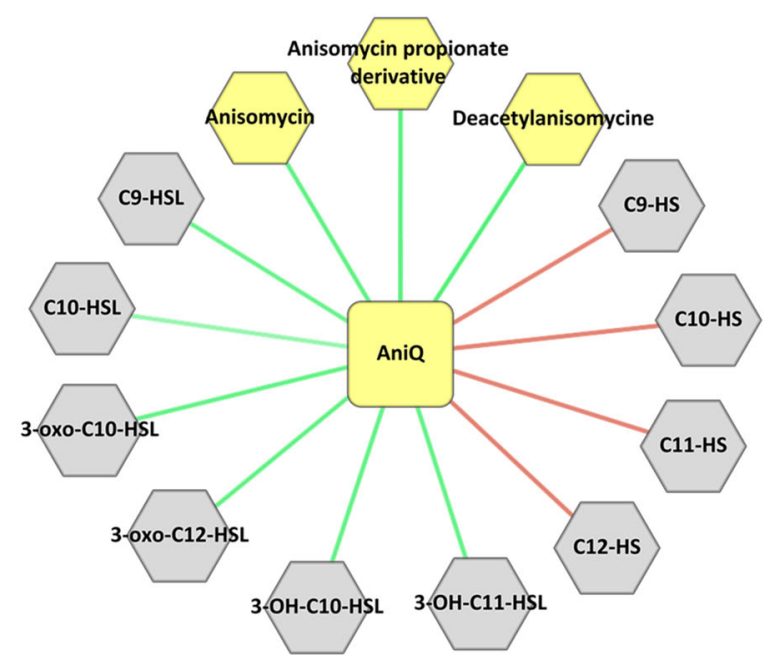

Fig. 7 Impact of SsoPox W263I on anisomycin biosynthesis in C. violaceum. a Organization of the anisomycin biosynthesis operon and impact of SsoPox W263I on the anisomycin biosynthetic pathway. In operon representation, arrows indicate the direction of transcription. Pathway and nomenclature are from Zheng et al. ${ }^{41}$. Proteins (dashed line boxes) and metabolites (full-line boxes) normalized scaled intensities (NSI) are represented as the mean and standard deviations (metabolomic: $n=3$ biological replicates, proteomic: $n=8,4$ biological replicates $\times 2$ technical replicates). ${ }^{*} P$ value $<0.05,{ }^{* *} P$ value $<0.01,{ }^{* * *} P$ value $<0.001$ according to Student's $t$ test. FC fold change. $\mathbf{b}$ Correlation multi-omics network of the protein AniQ (square) and metabolites (hexagons) involved in the production of anisomycin. Metabolites significantly impacted by SsoPox W263I are positively (green) or negatively (red) connected with AniQ.

SsoPox decreases C. violaceum toxicity in higher eukaryotic models: murine macrophages and planarians

C. violaceum is broadly found in water and soil microbiota in subtropical and tropical regions and has been recently identified as an environmental opportunistic pathogen infecting humans and animals ${ }^{49}$. This bacterium is thus known to be associated in rare, but frequently fatal, human infections underlining its high virulence under normal human body temperature ${ }^{50,51}$. To further probe the impact of SsoPox W263I on the virulence of CV 12472, a cytotoxicity model using macrophages was first developed (Fig. $8 \mathrm{c}$ ). The bacterial cytotoxicity at a multiplicity of infections (MOI) of
1, meaning a proportion of one bacterium for one macrophage, was assayed in $\mathbf{J 7 7 4 . 1}$ cells. Moderate cytotoxicity (30\%) was observed in this condition, which was used to study the enzymatic effect. Interestingly, upon enzymatic exposure, the cytotoxicity decreased down to $12 \%$, therefore suggesting a possible link between QS regulation and the observed cytotoxic effect. A similar inhibitory effect induced on the cytotoxicity of CV 12472 has previously been reported with guava leaf extract as the QS inhibitor in an infection model of HepG2 human liver carcinoma cells ${ }^{52}$. No cytotoxic effect was detected with supernatants of untreated or enzymatically treated CV 12472 cultures. These 


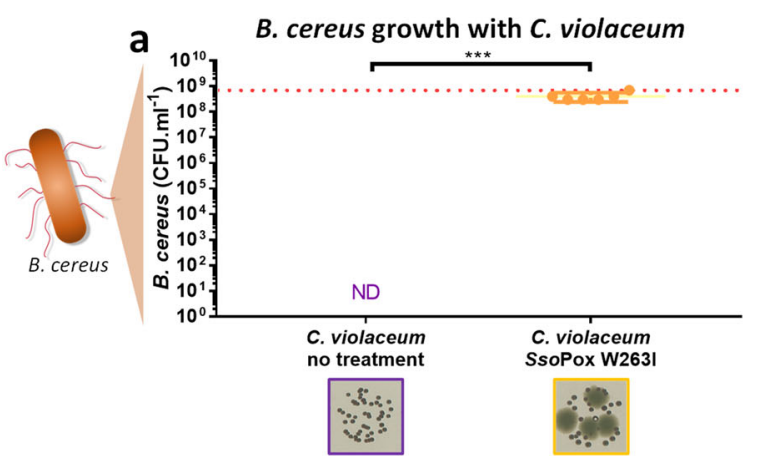

C

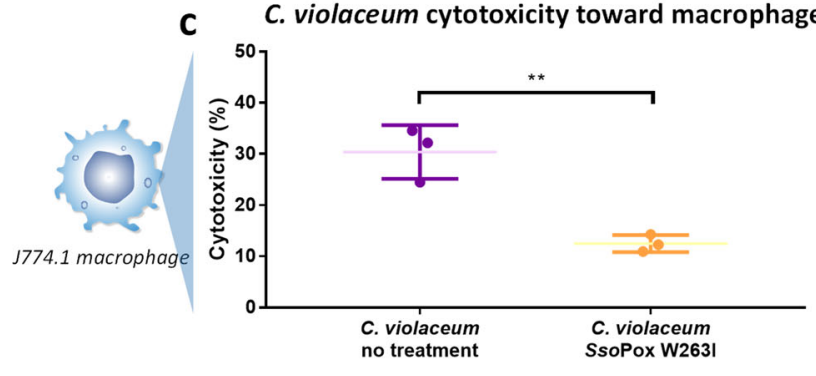

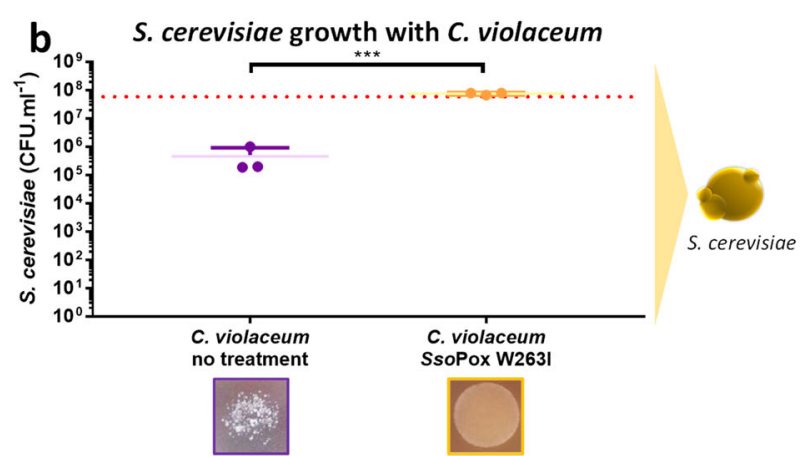

S. mediterranea survival with C. violaceum

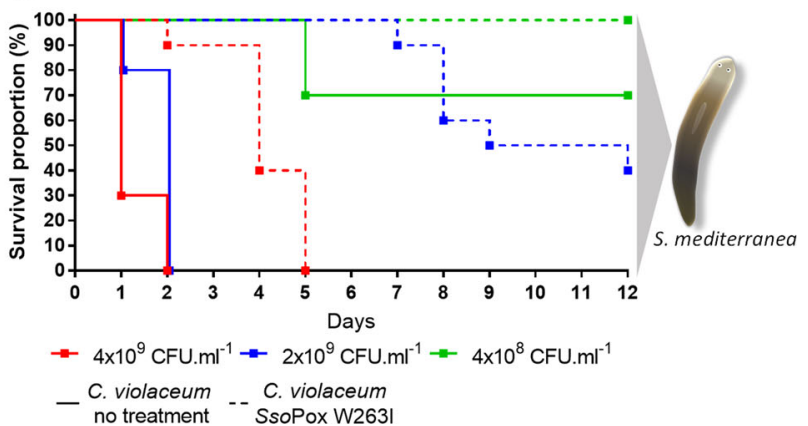

Fig. 8 SsoPox alters C. violaceum interaction with prokaryotic and eukaryotic organisms. a Mean bacterial concentration in $C F U \mathrm{ml}^{-1}$ of $B$. cereus after $8 \mathrm{~h}$ of cocultures with C. violaceum treated with $0.5 \mathrm{mg} \mathrm{ml}^{-1}$ SsoPox W263l (yellow) and untreated cultures as negative controls (purple). Error bars represent the standard deviations of $n=6$ experiments (three biological replicates $\times$ two technical replicates). The dotted red line represents the mean bacterial concentration of $B$. cereus monoculture. Representative pictures of dilution on LB-Agar plate (dilution factor at $10^{-6}$ ) of $B$. cereus cultures with treated cultures of $C$. violaceum with $0.5 \mathrm{mg} \mathrm{ml}^{-1}$ SsoPox W263I (yellow square) and untreated cultures as negative controls (purple square) are given. ${ }^{* * *} P$ values $<0.001$ according to the Student's $t$ test. ND not detected. b Mean yeast concentration in CFU ml ${ }^{-1}$ of S. cerevisiae after $24 \mathrm{~h}$ growth on YPD-Agar infected by C. violaceum treated with $0.5 \mathrm{mg} \mathrm{ml}^{-1}$ SsoPox W263I (yellow) and untreated as negative controls (purple). Error bars represent the standard deviations of $n=3$ biological replicates. The dotted red line represents the mean yeast concentration of S. cerevisiae on non-infected YPD-Agar. Representative pictures of dilution on YPD-Agar plate (dilution factor at $10^{-2}$ ) of $S$. cerevisiae growth on the plate infected by treated cultures of C. violaceum with $0.5 \mathrm{mg} \mathrm{ml}^{-1}$ SsoPox W263l (yellow square) and untreated cultures as negative controls (purple square) are given. ${ }^{* * *} P$ values $<0.001$ according to Student's $t$ test. ND not detected. c Cytotoxicity of $C$. violaceum toward 3774.1 macrophages with $0.5 \mathrm{mg} \mathrm{ml}^{-1}$ SsoPox W263l (yellow) or untreated as negative controls (purple) during a $1.5 \mathrm{~h}$ infection at a MOI of 1 . Error bars represent the standard deviations of $n=3$ replicated experiments. ${ }^{* * P}$ values $<0.01$ according to Student's $t$ test. d Planarian survival in the presence of $C$. violaceum treated with $0.5 \mathrm{mg} \mathrm{ml}^{-1}$ SsoPox W263I (dotted line) and untreated cultures as negative controls (full line) at $4 \times 10^{9} \mathrm{CFU} \mathrm{ml}^{-1}$ (red), $2 \times 10^{9} \mathrm{CFU} \mathrm{ml}^{-1}$ (blue), $4 \times 10^{8} \mathrm{CFU} \mathrm{ml}{ }^{-1}$ (green). Curves represent survival proportions of ten planarians. According to log-rank (Mantel-Cox) test comparing survival curves in treated and untreated conditions

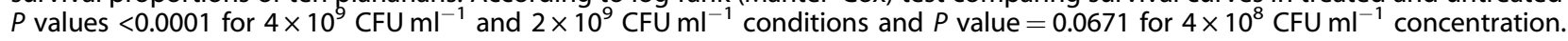

results indicated that cytotoxicity seemed independent from extracellular secretions, but rather implied some intracellular molecules or cellular mechanisms. We observed that SsoPox W263I reduced the production of CV_1744 protein (CV-IIL) (Fig. 2c), a fucophilic lectin, which resembles PA-IIL, a QS-regulated fucose-binding lectin of $P$. aeruginosa, known to be involved in bacterial adherence to host-cell in the initial steps of infections ${ }^{53,54}$. This lectin could then be involved in the reduction of the cytotoxicity of CV 12472. As AHLs, and more particularly 3-oxoC12-HSL, harbor an important toxic activity toward eukaryotic cells ${ }^{55}$, the degradation of these molecules induced by SsoPox W263I could also play a role in the reduction of the cytotoxic effect induced by CV 12472. The decrease of CV 12472 toxicity towards macrophages upon lactonase treatment suggested that $\mathrm{QQ}$ is a promising alternative approach to tackle human and animal infections by $C$. violaceum.

In C. violaceum, QS regulates the biosynthesis of toxic metabolites, including violacein, hydrogen cyanide, and anisomycin. To assess whether QS may contribute to $C$. violaceum competition with animals, we used an aquatic model organism, the freshwater flatworm Schmidtea mediterranea. In recent decades, planarians have emerged as relevant invertebrate models for toxicology and ecotoxicology studies and have been used to evaluate neurodegenerative diseases as well as the toxicity of environmental pollutants and neurotoxic chemicals ${ }^{56-59}$. Moreover, planarians have been previously described as particularly resistant to bacterial infections by eliminating bacteria mainly through phagocytosis ${ }^{60}$. Here, we used lifespan assay to evaluate the toxicity of $C$. violaceum on S. mediterranea. Bacteria were first grown with or without lactonase and then incubated with planarians. Planarians were not able to eliminate the bacterial threat in either of these conditions as the bacterial concentrations remained equivalent to those of the worm-free control for 12 days (Supplementary Fig. 6). We showed that $C$. violaceum rapidly kills planarians from a bacterial load of $4 \times 10^{9} \mathrm{CFU} \mathrm{ml}^{-1}$ (Fig. 8d). Interestingly, lactonase-mediated quenching decreased bacterial toxicity and significantly enhanced worms' survival, leading to complete inhibition of mortality at $4 \times 10^{8} \mathrm{CFU} \mathrm{ml}^{-1}$.

These results underscored that $\mathrm{QS}$ can provide $C$. violaceum important advantages for environmental interactions, such as better fitness when competing against animals as planarians. In previous studies, violacein has been described for its nematicide effect against Caenorhabditis elegans ${ }^{61,62}$ and anisomycin, a translation inhibitor in eukaryotes has been shown to affect wound healing in planarians ${ }^{63}$ but other factors might also be involved in toxicity towards planarians. Consistently, we showed that QS mediates the toxicity of $C$. violaceum in $S$. mediterranea and that enzymatic quenching can reduce the virulence of this 
bacterial strain toward planarians. The toxic mechanisms produced by CV 12472 and killing planarians remain unknown but they probably differ from other toxic models that require bacterial ingestion since the bacterial load remains unchanged along the experiment and that cell-free supernatants also showed toxicity (Supplementary Fig. 7).

\section{DISCUSSION}

QS disruption driven by lactonase SsoPox W263I was previously shown to modulate microbial communities from soil and lake water samples thus affecting ecological behaviors including biofilm formation, colonization or biocorrosion. To further address the direct and indirect impacts of this enzyme on diverse bacteria able or not to produce or sense AHLs, we evaluated the molecular and phenotypic mechanisms involved in social interactions of CV 12472 , a proteobacterium representative from natural environments.

The production of the main AHLs of CV 12472, namely C10-HSL and $3-\mathrm{OH}-\mathrm{C} 10-\mathrm{HSL}$ was first confirmed, along with $\mathrm{C} 9-\mathrm{HSL}, \mathrm{C} 11-$ $\mathrm{HSL}$, 3-OH-C11-HSL, 3-oxo-C10-HSL, 3-oxo-C12-HSL which had never been described before in this strain. All AHLs were present both in whole-cell extracts and cell-free supernatants. SsoPox W263I quenching effect was confirmed by the diminution of the production of these AHLs in treated samples and the detection of the hydrolyzed forms of some of them (C9-HS, C10-HS, C11-HS, and C12-HS, respectively).

Common QS-regulated traits, such as exoprotease production and biofilm formation, were shown to be inhibited by the enzymatic treatment. Moreover, SsoPox W263I also induced a decrease in the production of both hydrogen cyanide and violacein observed at proteomic, metabolomic, and phenotypic levels.

The biosynthesis of anisomycin was unexpectedly detected in CV 12472 (in whole-cell and cell-free supernatant extracts) and the presence of the corresponding biosynthetic gene cluster (only previously hypothesized) was confirmed. A downregulated impact of the QQ treatment was observed for one protein involved in anisomycin biosynthesis, as well as the decrease of the final metabolites of this biosynthetic pathway, showing that anisomycin production is under QS regulation in this strain.

Considering the broad impact of QS on the synthesis of toxic metabolites as well as the impact of enzymatic QQ (Supplementary Fig. 8), the effect of the lactonase SsoPox W263I on C. violaceum social interactions was evaluated in prokaryotic and eukaryotic competition assays. Treatment of CV 12472 by the lactonase was found to drastically inhibit toxicity toward a Grampositive bacterium, yeast, and higher eukaryotic organisms using in vitro and in vivo models. Competition models with $B$. cereus and $S$. cerevisiae revealed that treatment with the $\mathrm{QQ}$ agent in $C$. violaceum decreased the production of extracellular antimicrobial compounds. We found that CV 12472 toxic effect on B. cereus was mainly ascribable to violacein and that anisomycin affected the morphology of $S$. cerevisiae, while its sole action did not alter yeast growth.

Besides its impact on interactions with bacteria and yeast, lactonase treatment was found to decrease CV 12472 cytotoxicity toward J774.1 murine macrophages and reduce mortality in planarians. These investigations confirmed the toxicity of CV 12472 in higher eukaryotic models and suggested that QS may be a relevant target to decrease $C$. violaceum toxicity towards higher eukaryotes.

This study draws a complete overview of the ability of lactonase-mediated $\mathrm{QQ}$ to disrupt bacterial competition toward other organisms. Under our experimental conditions, the effects of SsoPox W263I observed on CV 12472 cultures provide essential insights into the role of QS in interkingdom relations and pave the way for further investigations, particularly concerning the role of
QQE on bacterial populations. Here, the results obtained by combining molecular analyses and simplistic competitions assays showed evidence that lactonase-mediated QS disruption may affect bacteria in both inter- and intra-kingdom interactions. Complex environments and interactions are hard to reproduce under laboratory conditions due to challenges in combining different organisms' growth requirements. To fully understand the role of QS in multispecies communities and dynamics, metagenomic data would need to be examined in light of metabolomic analyses to identify the molecular causes of microscopic changes and population dynamics.

\section{METHODS}

\section{Microbial strains and growth conditions}

Experiments were conducted using Chromobacterium violaceum ATCC 12472, Bacillus cereus ATCC 1457, and Saccharomyces cerevisiae ATCC 9763. Bacteria were grown in Luria Bertani (LB) medium $\left(10 \mathrm{gl}^{-1} \mathrm{NaCl}, 10 \mathrm{gl}^{-1}\right.$ tryptone, $5 \mathrm{gl}^{-1}$ yeast extract) at $37^{\circ} \mathrm{C}$ and yeast were grown in Yeast extract-Peptone-Dextrose (YPD) medium $\left(10 \mathrm{gl}^{-1}\right.$ yeast extract, $20 \mathrm{gl}^{-1}$ peptone, $10 \mathrm{gl}^{-1}$ glucose) at $30^{\circ} \mathrm{C}$. For C. violaceum, bacteria were inoculated from a single colony and pre-cultivated for $6 \mathrm{~h}$ at $37^{\circ} \mathrm{C}$ in LB medium with agitation at $350 \mathrm{rpm}$. Then, precultures were diluted by a 1 000 factor in LB medium in 12-well plates, and cultures were incubated at $37^{\circ} \mathrm{C}$ under agitation at $350 \mathrm{rpm}$ for $16 \mathrm{~h}$. The enzyme was added, when indicated, at a final concentration of $0.5 \mathrm{mg} \mathrm{ml}^{-1}$.

\section{Protein production and purification}

SsoPox W263I variant was used as QQ enzyme and productions were performed as follow ${ }^{18,64,65}$. Briefly, cells of Escherichia coli Bl21 (DE3)pGro7/GroEL (TaKaRa Bio, Shiga, Japan), carrying plasmid pET22b-SsoPoxW263I, were cultivated in ZYP-5052 medium complemented with $100 \mu \mathrm{g}$ $\mathrm{ml}^{-1}$ ampicillin and $34 \mathrm{\mu g} \mathrm{m}^{-1}$ chloramphenicol at $37^{\circ} \mathrm{C}$ to a final OD $600 \mathrm{~nm}$ of $0.8-1$. L-arabinose was added to induce the expression of chaperone proteins at a final concentration of $0.2 \%(\mathrm{w} / \mathrm{v})$. Simultaneously, $0.2 \mathrm{mM}$ of $\mathrm{CoCl}_{2}$ was added, and the temperature was reduced down to $23^{\circ} \mathrm{C}$. Cells were harvested by centrifugation $\left(4400 \times g, 4^{\circ} \mathrm{C}, 20 \mathrm{~min}\right)$ after $20 \mathrm{~h}$ of incubation. The pellet was then resuspended in lysis buffer $(50 \mathrm{mM}$ HEPES pH 8, $150 \mathrm{mM} \mathrm{NaCl} 0.25 \mathrm{mg} \mathrm{m}^{-1}$ lysozyme, $0.1 \mathrm{mM}$ phenylmethylsulfonyl fluoride (PMSF), and $10 \mathrm{\mu g} \mathrm{ml}^{-1}$ DNAsel) and stored at $-80^{\circ} \mathrm{C}$ for $16 \mathrm{~h}$. Cells were thawed for $15 \mathrm{~min}$ at $37^{\circ} \mathrm{C}$ and lysed by three steps of $30 \mathrm{~s}$ sonication (QSonica sonicator Q700, amplitude at 45). Centrifugation was used to remove cell debris $\left(21,000 \times g, 4^{\circ} \mathrm{C}, 20 \mathrm{~min}\right)$. The resulting extract was incubated for $30 \mathrm{~min}$ at $80^{\circ} \mathrm{C}$ and centrifuged to remove the precipitate $\left(21,000 \times g, 4^{\circ} \mathrm{C}, 30 \mathrm{~min}\right)$. The enzyme was concentrated by overnight incubation at $4{ }^{\circ} \mathrm{C}$ in $75 \%$ ammonium sulfate. After resuspension in activity buffer ( $50 \mathrm{mM}$ HEPES $\mathrm{pH} 8,150 \mathrm{mM} \mathrm{NaCl}$ ), ammonium sulfate was eliminated by desalting step (HiPrep 26/10 desalting, GE Healthcare, ÄKTA Avant, Chicago, IL, USA). The protein sample was concentrated using $30 \mathrm{kDa}$ centricon, and then purified to homogeneity using a size-exclusion chromatography column (Hiload 16/600 Superdex ${ }^{\mathrm{TM}} 75$ pg, GE Healthcare, ÄKTA Avant). Protein purity was checked by migration on $10 \%$ SDS-PAGE, and protein concentration was measured using a spectrophotometer NanoDrop 2000 (Thermo Fisher Scientific, Waltham, MA, USA).

\section{Proteome extraction}

After $16 \mathrm{~h}$ of culture in LB with or without enzyme, bacteria were collected. The samples were centrifuged $5 \mathrm{~min}$ at $11,000 \times g$, the supernatant was discarded, and the pellet was washed twice in phosphate-buffered saline solution (PBS). To prepare total protein samples, the supernatant was discarded and the pellet was resuspended in UTS buffer ( $8 \mathrm{M}$ urea, $2 \mathrm{M}$ thiourea, $100 \mathrm{mM} \mathrm{NaCl}, 25 \mathrm{mM}$ Tris, $\mathrm{pH}$ 8.0, and protease inhibitors (Roche, Pleasanton, CA, USA, Complete, EDTA free)). The samples were sonicated three times for $1 \mathrm{~min}$ (QSonica Q700, amplitude at 45 with $30 \mathrm{~s}$ intermittence) and were then centrifuged at $13,000 \times g$ for $30 \mathrm{~min}$ at room temperature. In a second time, the supernatant was transferred in dialysis cassettes (Slide-A-lyser Dialysis Cassettes 2 K MWCO, Thermo Fisher Scientific) and placed in 21 of Urea/Ambic buffer (1 M urea, $50 \mathrm{mM}$ ammonium bicarbonate) for $4 \mathrm{~h}$ followed by one night in $2 \mathrm{l}$ of fresh Urea/ Ambic buffer. The protein concentration in each sample was then determined using Bradford's assay. Samples containing $50 \mu \mathrm{g}$ of proteins were prepared to a final volume of $45 \mu \mathrm{l}$ using Urea/Ambic buffer. For 
disulfide bond reduction, $1 \mu \mathrm{l}$ of DTT (0.5 M dithiothreitol in Urea/Ambic buffer) was added and the samples were incubated for $1 \mathrm{~h}$ at $30^{\circ} \mathrm{C}$. For alkylation, $2 \mu \mathrm{l}$ of IAA ( $0.5 \mathrm{M}$ iodoacetamide in Urea/Ambic buffer) was then added before a $1 \mathrm{~h}$ incubation in the dark. Proteins were then digested by adding $2 \mu \mathrm{l}$ of trypsin for a ratio 1:25 (w:W), and the samples were incubated overnight at $37^{\circ} \mathrm{C}$. Finally, to remove detergents and clean the samples, Pierce detergent removal spin column (PierceTM, Thermo Fisher Scientific) and C18 spin column (PierceTM) were used according to the manufacturer's recommendations. The result of this preparation was suspended in acetonitrile $70 \%$. The solutions were evaporated and resolubilized in $50 \mu \mathrm{l}$ of eluent $(0.1 \%$ formic acid) to have $1 \mu \mathrm{g} / 1 \mu \mathrm{l}$ solutions of peptides.

\section{Label-free quantitative nano-LC-MS/MS proteomic analysis}

Protein digests were first separated by ultraperformance liquid chromatography (UPLC) using the NanoAcquity UPLC System (Waters, Elstree, UK) connected to a Synapt G2Si Q-TOF ion mobility hybrid mass spectrometer (Waters). The chromatographic system was used in 1D configuration with an analytical column (ACQUITY UPLC M-Class peptide CSH C18 Column, $130 \AA 1.7 \mu \mathrm{m}, 75 \mu \mathrm{m} \times 100 \mathrm{~mm}$, Waters) after a trapping column (ACQUITY UPLC M-Class Symmetry C18 Trap Column, $100 \AA 5 \mu \mathrm{m} 2 \mathrm{G} \mathrm{V} / \mathrm{M}, 180 \mu \mathrm{m} \times$ $20 \mathrm{~mm}$, Waters). Eluted peptides were then separated using a $100 \mathrm{~min}$ gradient ( $300 \mathrm{nl} \mathrm{min}{ }^{-1} ; 0.5$ to $40 \%$ acetonitrile- $0.1 \%$ formic acid). Dataindependent MS/MS analysis was performed with the ion mobility feature (HDMSe method). The parameters of the ion source were capillary voltage at $3 \mathrm{kV}$, sampling cone voltage at $40 \mathrm{~V}$, ion source temperature at $90^{\circ} \mathrm{C}$, cone gas flow at $50 \mathrm{I} \mathrm{h}^{-1}$. Transfer collision low energy was set to $5 \mathrm{~V}$, trap collision low energy was set to $4 \mathrm{~V}$. The high energy ramp was applied from 4 to $5 \mathrm{~V}$ for the trap collision and from 19 to $45 \mathrm{~V}$ for the transfer collision enabling fragmentation of the ions after ion mobility cell and before the time-of-flight (TOF) MS. The On-column sample load was $800 \mathrm{ng}$. Each sample was injected in duplicate.

The files acquired were imported into Progenesis QI software v. 2.0 (Nonlinear Dynamics, Newcastle, UK) for label-free quantification analysis. The data were automatically aligned and normalized. Processing parameters were 150 counts for the low energy threshold, 30 counts for the elevated energy threshold. The database used to identify peptides contains the protein sequences of C. violaceum ATCC 12472 (UniProt, 08/ 05/2018, 4397 sequences). Search tolerance parameters were as follows: peptide and fragment tolerance, $15 \mathrm{ppm}, \mathrm{FDR}<1 \%$; minimum ion matching requirements were three fragments per peptide, seven fragments per protein, and two peptides per protein. The enzyme specificity was trypsin allowing one missed cleavage. The accepted modifications were carbamidomethyl of cysteine (fixed), oxidation of methionine (variable), carbamyl of lysine and $\mathrm{N}$-terminal (variable), deamidation (variable) of asparagine and glutamine. The protein normalization was performed according to the relative quantitation using nonconflicting peptides. To determine the significance of changes between samples, a significant ANOVA ( $P$ value $<0.05$ ) and a fold change superior to 1.5 were used as thresholds to define differently expressed proteins.

Functional annotation of the differentially expressed proteins was carried out using DAVID annotation tool (https://david.ncifcrf.gov/), the gene ontology (GO) database in the UniProt Knowledgebase (UniProtKB) and bibliography. Of note, as $C$. violaceum is known to be highly virulent at normal human body temperature, we chose to grow the bacterium at $37^{\circ} \mathrm{C}$ in all our experiments. This must be considered when comparing our "omics" results with previously reported data mainly performed at $30^{\circ} \mathrm{C}$.

\section{Metabolite extraction}

In total, $25 \mathrm{ml}$ of bacterial culture (triplicates) in LB with or without enzyme were extracted using $5 \mathrm{ml}$ of ethyl acetate (LC-MS grade, Sigma-Aldrich, St. Louis, MO, USA). After 30 min of sonication, the upper organic phase was collected and dried in vacuo. The resulting dried extracts were solubilized in methanol (LC-MS grade, Sigma-Aldrich), filtered $(0.2 \mu \mathrm{m})$, dried under a nitrogen flow, and were then kept in nitrogen at $-20^{\circ} \mathrm{C}$. For more details, see ref. ${ }^{66}$. For supernatant extraction, $25 \mathrm{ml}$ of bacterial cultures in LB were centrifuged, the recovered supernatant was filtered $(0.22 \mu \mathrm{m})$ and extracted using $5 \mathrm{ml}$ of ethyl acetate. The upper organic phase was collected and the same procedure was applied as for whole-culture extractions.

\section{LC-ESI-MS and MS/MS data acquisition}

Samples were prepared by solubilizing the dry extracts in $1 \mathrm{ml}$ of LC-MS grade methanol. Six medium blanks were also prepared using the same protocol but without bacteria. Four quality control samples (QCs) were prepared by mixing an aliquot of all the samples. Samples and medium blanks were randomly injected, and a QC was injected every three samples. At the beginning of the injection sequence, two analytical blanks (only methanol) and a QC were injected before the samples.

Samples were analyzed by UPLC-ESI-QToF-MS in positive mode. The UPLC-MS instrumentation consisted of a Dionex Ultimate 3000 Rapid Separation (Thermo Fisher Scientific) chromatographic system coupled with a QToF Impact II mass spectrometer (Bruker Daltonics, Mannheim, Germany). The separations were carried out on a reverse-phase column $(150 \times 2.1 \mathrm{~mm}, 1.7 \mu \mathrm{m}$, Kinetex Phenyl-Hexyl; Phenomenex, Torrance, CA, USA) equipped with a pre-column (SecurityGuard cartridge, Phenomenex) at a temperature of $40^{\circ} \mathrm{C}$. The injection volume was $5 \mu$, and the flow rate was set at $0.5 \mathrm{ml} \mathrm{min}-1$. The autosampler temperature was set at $4{ }^{\circ} \mathrm{C}$, and the injection volume was $5 \mu$ l. Mobile phases were: (A) water and (B) acetonitrile (Chromasolv; Sigma-Aldrich) containing each $0.1 \%(\mathrm{v} / \mathrm{v})$ of formic acid (ultra grade; Fluka, Fischer Scientific). The elution gradient started at $5 \%$ B, maintained for 2 min, then increased to $100 \%$ B (linear ramp) in $8 \mathrm{~min}$, and maintained for $4 \mathrm{~min}$; then back to $5 \% \mathrm{~B}$ (linear ramp) over $0.01 \mathrm{~min}$ and maintained $1.99 \mathrm{~min}$, for a total run time of $16 \mathrm{~min}$.

The capillary voltage of the MS spectrometer was set at $4500 \mathrm{~V}$ (positive mode), and the nebulizing parameters were set as follows: nebulizing gas $\left(\mathrm{N}_{2}\right)$ pressure at $0.4 \mathrm{bar}$, drying gas $\left(\mathrm{N}_{2}\right)$ flow at $4 \mathrm{Imin}^{-1}$, and drying temperature at $180^{\circ} \mathrm{C}$. Mass spectra were recorded from $\mathrm{m} / \mathrm{z} 50$ to 1200 at a mass resolving power of 25,000 full width at half-maximum (FWHM, $\mathrm{m} / \mathrm{z}$ $=200$ ) and a frequency of $2 \mathrm{~Hz}$. Tandem mass spectrometry analyses were performed thanks to collision-induced dissociation (CID) with a collision energy of $25 \mathrm{eV}$. A solution of formate/acetate forming clusters was automatically injected before each sample for internal mass calibration, and the mass spectrometer was calibrated with the same solution at the beginning of the sequence.

\section{Metabolomic data processing and analysis}

LC-MS raw data were converted into ".netCDF" files using DataAnalysis v. 4.3 (Bruker, Mannheim, Germany) and processed with MZmine v. $2.53^{67}$. All steps and parameters used to obtain the final data matrix are listed in Supplementary Table 1. In a second time, the data were submitted to two filtering steps using an in-house script on $\mathrm{R}$ and final manual filtering. Filtering consisted in removing successively experimental and analytical bias according to signal/noise ratio (using blanks) and coefficient of variation (using QCs). For multivariate analyses, the resulting data matrix was $\log _{10}$-transformed, mean-centered, and analyzed using MetaboAnalyst 4.5 online $^{68}$. Data were analyzed using Principal Component Analysis (PCA) and Partial Least Squares (PLS-DA) discriminant analysis. From the PLS-DA, the most discriminating $\mathrm{m} / \mathrm{z}$ features (VIPs) were selected according to their VIP score. This statistical model was evaluated using a permutation test and a cross-validation analysis of variance (MetaboAnalyst 4.5 online) The comparison of metabolites of interest between the two culture conditions was carried out using a $t$ test (GraphPad Prism v. 7.04).

\section{Feature annotation via molecular networking approach}

LC-MS/MS raw data (samples and standards) were converted into ".mzXML" files using DataAnalysis. The Internet platform GNPS (http:// gnps.ucsd.edu) was used to create molecular networking of related compounds based on the similarity of their MS/MS fragmentation patterns ${ }^{23}$. Similar chemical structures share similar fragmentation pathways in mass spectrometry leading to identical fragments and losses ${ }^{69}$. The parameters used were: Precursor lon Mass Tolerance (Da): 0.02, Fragment Ion Mass Tolerance $(\mathrm{Da})$ : 0.02 , Minimum Cosine Score: 0.62, Minimum Matched Fragment lons: 4 and Network TopK: 10. Data were imported and treated offline (Cystoscape $v$ 3.7.0). Two methods were used for the annotation of a specific $\mathrm{m} / \mathrm{z}$ feature: (1) Searching the most probable molecular formula with DataAnalysis software with the "Smartformula" package, (2) Using online databases, such as PubChem (https://pubchem. ncbi.nlm.nih.gov), Lipidmaps (http://www.lipidmaps.org), CEU mass mediator (http://ceumass.eps.uspceu.es), Metlin (https://metlin.scripps.edu) ${ }^{70}$, or SIRIUS $4.4 .17^{71}$. Finally, a careful analysis of the MS/MS fragmentation pattern in comparison with the literature data was performed to confirm the putative annotation. 


\section{Multi-omic data processing}

Proteomic and metabolomic data matrices were processed by regularized Canonical Correlation Analysis ( $\mathrm{rCCA})^{34-36}$ with the R package MixOmics following the protocol found on the website http://mixomics.org/. The shrinkage method was used to adjust the regularization parameters $\lambda 1$ and $\lambda 2$. A cut-off value of 0.5 was chosen to create a molecular network with the function write.graph() under the format ".graphml". The resulting network was exported and treated using Cytoscape v. 3.7.0.

\section{Lactonase activity measurement}

The activity was measured on C9-HSL,C10-HSL, C11-HSL, C12-HSL, and 3$\mathrm{OH}-\mathrm{C} 10-\mathrm{HSL}$ at ambient temperature using a colorimetric $\mathrm{pH}$-based assay $^{72}$. Briefly, the hydrolysis of the lactone ring leads to the acidification of the solution, which is followed by the absorbance modification of a pH indicator (cresol). For kinetic parameters, the degradation of lactones at different concentrations by each enzyme in a cresol-buffered solution (1.25 mM Bicine, $150 \mathrm{mM} \mathrm{NaCl}, 0.2 \mathrm{mM} \mathrm{CoCl}, 0.25 \mathrm{mM}$ cresol purple, $3.5 \%$ for C9-HSL, C11-HSL, and C12-HSL or a minimum of $1.5 \%$ DMSO for C10HSL and 3-OH-C10-HSL, and pH 8.3) was followed in $200 \mu$ at $577 \mathrm{~nm}$ using a plate reader (Synergy HT, BioTek, Winooski, VT, USA). For C9-HSL and C11-HSL, $10 \mathrm{mg} \mathrm{ml}^{-1}$ of SsoPox W263I were used, and $20 \mathrm{mg} \mathrm{ml}^{-1}$ for C10HSL, C12-HSL, and 3-OH-C10-HSL. The activity of SsoPox W263I was not assayed on $3-\mathrm{OH}-\mathrm{C} 11-\mathrm{HSL}$ due to its commercial unavailability.

\section{Analysis of quorum sensing-regulated factors}

QS-regulated factor productions were determined in vitro after $16 \mathrm{~h}$ of culture at $37^{\circ} \mathrm{C}$ with agitation (350 rpm) in the presence of $0.5 \mathrm{mg} \mathrm{ml}^{-1}$ SsoPox W263I and without enzyme.

\section{Biofilm formation}

Biofilm was measured using crystal violet ${ }^{73}$. Planktonic cells were removed, and wells were washed once with PBS. After drying the plates at $37^{\circ} \mathrm{C}$ until complete evaporation of PBS, crystal violet $0.5 \%$ was added, and following a wash with PBS, the remaining colorant was dissolved using ethanol $100 \%$. Then, $200 \mu \mathrm{l}$ were collected and absorbance was measured at $595 \mathrm{~nm}$ using a microplate reader (Synergy HT, BioTek, Winooski, VT, USA).

\section{Proteolytic activity}

Cell-free culture supernatants were prepared by centrifugation $(10 \mathrm{~min}$ $12,000 \times g$, room temperature). Protease activity was measured using azocasein (Sigma-Aldrich) ${ }^{74}$. Then $25 \mu$ l of cell-free supernatant was added to a mixed solution of $675 \mu \mathrm{l}$ PBS solution pH 7 and $50 \mu \mathrm{l}$ of azocasein $\left(30 \mathrm{mg} \mathrm{ml}^{-1}\right.$ in water). Samples were incubated for $2 \mathrm{~h}$ at $37^{\circ} \mathrm{C}$, the reaction was then stopped by adding $125 \mu \mathrm{l}$ of $20 \%(\mathrm{w} / \mathrm{v})$ trichloroacetic acid. The samples were centrifuged $(10 \mathrm{~min}, 10,000 \times g$, room temperature), and the absorbance of $200 \mu \mathrm{l}$ of supernatant was measured at $366 \mathrm{~nm}$ using a plate reader (Synergy HT, BioTek).

\section{Violacein production}

Violacein was extracted from $500 \mu \mathrm{l}$ of cell-free supernatant by adding $500 \mu \mathrm{l}$ of ethyl acetate ${ }^{75}$, the mixture was vortexed then centrifuged ( $5 \mathrm{~min}$, $10,000 \times g$, room temperature), and $200 \mu \mathrm{l}$ of the upper phase was collected. The absorbance was measured at $565 \mathrm{~nm}$ using a microplate reader (Synergy HT, BioTek).

To estimate the concentration of violacein in the supernatant, $\mathrm{OD}_{565}$ values were compared to a standard curve made with pure violacein isolated from C. violaceum (Coger, Paris, France) resuspended in LB at a known concentration ranging from 1 to $40 \mu \mathrm{g} \mathrm{ml}^{-1}$.

\section{Hydrogen cyanide production}

Extracellular cyanide production was measured by using methemoglo$\mathrm{bin}^{76}$. The formation of cyanomethemoglobin complex induces a shift in the maximum absorbance spectrum from $407 \mathrm{~nm}$ (free methemoglobin) to $424 \mathrm{~nm}$ (cyanomethemoglobin complex). Methemoglobin reagent was prepared by dissolving $85 \mathrm{mg}$ of bovine hemoglobin in $12.5 \mathrm{ml}$ of $\mathrm{NaNO}_{2}$, then $12.5 \mathrm{ml}$ of phosphate buffer $0.1 \mathrm{M}$ were added to the methemoglobin reagent. In total, $50 \mu \mathrm{l}$ of cell-free supernatants were diluted with $140 \mu \mathrm{l}$ of Millipore water. The absorbance background of the samples was measured at $424 \mathrm{~nm}$ using a plate reader (Synergy HT, BioTek). Subsequently, $10 \mu \mathrm{l}$ of methemoglobin reagent was added to give a final volume of $200 \mu \mathrm{l}$ and let react for $30 \mathrm{~min}$. Finally, the absorbance of the samples was measured at $424 \mathrm{~nm}$. The absorbance background was then subtracted from the final measure.

To estimate the concentration of hydrogen cyanide in the supernatant, $\mathrm{OD}_{424}$ values were compared to a standard curve realized with potassium cyanide (Sigma) resuspended in LB at a known concentration ranging from 0.5 to $8 \mu \mathrm{g} \mathrm{ml}^{-1}$.

\section{Competition assay with $B$. cereus}

After $16 \mathrm{~h}$ culture in LB with or without enzyme for $C$. violaceum and an overnight preculture $(16 \mathrm{~h})$ in $\mathrm{LB}$ at $37^{\circ} \mathrm{C}$ with agitation for $B$. cereus, bacteria were inoculated at $1 / 100$ in LB medium. The cocultures were incubated at $37^{\circ} \mathrm{C}$ under agitation at $350 \mathrm{rpm}$ for $8 \mathrm{~h}$. Then the samples were serially diluted and plated on an LB-Agar plate. Colony-forming units (CFUs) were counted after overnight incubation at $37^{\circ} \mathrm{C}$.

\section{Bactericidal effect of $C$. violaceum supernatant against $B$. cereus}

To evaluate the bactericidal effect of $C$. violaceum supernatant against $B$. cereus, supernatant from a $16 \mathrm{~h}$ culture in LB with or without enzyme of $C$. violaceum was collected by centrifugation and filtered at $0.22 \mu \mathrm{m}$. An overnight preculture of $B$. cereus was inoculated at $1 / 1000$ corresponding to $3 \times 10^{5} \mathrm{CFU} \mathrm{ml}^{-1}$ in LB with $50 \%$ of $C$. violaceum supernatant. After an incubation of $8 \mathrm{~h}$ at $37^{\circ} \mathrm{C}$ with agitation at $350 \mathrm{rpm}$, the culture was serially diluted and plated on LB-Agar plates. After a night at $37^{\circ} \mathrm{C}$, CFUs were counted.

\section{Toxicity effect of hydrogen cyanide and violacein against $B$. cereus}

To evaluate the toxic effect of hydrogen cyanide and violacein supernatant against B. cereus, KCN (Sigma-Aldrich) and violacein (Coger) were resuspended in $\mathrm{LB}$ at the required concentration. An overnight preculture of $B$. cereus was inoculated at $1 / 1000\left(3 \times 10^{5} \mathrm{CFU} \mathrm{ml}^{-1}\right)$ in LB with $50 \%$ of $\mathrm{KCN}$ or violacein solution. After an incubation of $8 \mathrm{~h}$ at $37^{\circ} \mathrm{C}$ with agitation at $350 \mathrm{rpm}$, the culture was serially diluted and plated on LB-Agar plates. After $16 \mathrm{~h}$ at $37^{\circ} \mathrm{C}$, CFUs were counted.

Antifungal activity of $C$. violaceum toward S. cerevisiae assay To evaluate the toxic effect of $C$. violaceum on $S$. cerevisiae growth, $1.5 \mathrm{ml}$ of a $16 \mathrm{~h}$ culture with or without enzyme of $C$. violaceum were collected and centrifuged for $5 \mathrm{~min}$ at $10,000 \times g$. Supernatants were discarded and pellets were washed with PBS buffer. After a centrifugation step $(10,000 \times g$, $5 \mathrm{~min}$, room temperature), the pellets were resuspended in $1.5 \mathrm{ml}$ of melted YPD-Agar (10 $\mathrm{gl}^{-1}$ yeast extract, $20 \mathrm{gl}^{-1}$ peptone, $10 \mathrm{gl}^{-1}$ glucose, $1.5 \%$ agar) and poured in a 12-well plate. S. cerevisiae was inoculated from a single colony and pre-cultivated during $16 \mathrm{~h}$ at $30^{\circ} \mathrm{C}$ in YPD medium with agitation at $350 \mathrm{rpm}$. In total, $10 \mu \mathrm{l}$ of these cultures and tenfold serial dilutions were spotted onto $C$. violaceum inoculated YPD-Agar. After $16 \mathrm{~h}$ at $30^{\circ} \mathrm{C}$, CFUs were counted.

\section{Toxicity effect of $C$. violaceum supernatants and anisomycin against $S$. cerevisiae}

To evaluate the bactericidal effect of $C$. violaceum cell-free supernatants against $S$. cerevisiae, supernatants from a $16 \mathrm{~h}$ culture in LB with and without enzyme of $C$. violaceum were collected by centrifugation and filtered at $0.22 \mu \mathrm{m}$. The supernatants were added at a final concentration of $50 \%(\mathrm{v} / \mathrm{v})$ in YPD-Agar $1.5 \%$ at $56^{\circ} \mathrm{C}$ and poured in a 12 -well plate. In all, $10 \mu \mathrm{l}$ of an overnight preculture of S. cerevisiae and tenfold serial dilutions were spotted on anisomycin-containing YPD-Agar. After $16 \mathrm{~h}$ growth at $30{ }^{\circ} \mathrm{C}$, S. cerevisiae CFUs were counted.

\section{Toxicity effect of anisomycin against $S$. cerevisiae}

To evaluate the toxic effect of anisomycin against $S$. cerevisiae, anisomycin from Streptomyces griseolus (Sigma-Aldrich) was added in a concentration ranging from $50 \mathrm{nM}$ to $500 \mu \mathrm{M}$ in YPD-Agar $1.5 \%$ at $56^{\circ} \mathrm{C}$ and poured in a 12-well plate. In total, $10 \mu \mathrm{l}$ of an overnight preculture of $\mathrm{S}$. cerevisiae and tenfold serial dilutions were spotted on anisomycin-containing YPD-Agar. After $16 \mathrm{~h}$ at $30^{\circ} \mathrm{C}$, CFUs were counted. 


\section{LDH cytotoxicity assay toward J774.1 macrophages}

J774.1 macrophages (murine macrophage) were grown in $75 \mathrm{~cm}^{2}$ ventilated flask in Roswell Park Memorial Institute (RPMI) 1640 Medium Gibco $^{\text {TM }}$ (Thermo Fischer Scientific, Waltham, MA, USA) supplemented with $10 \%$ fetal bovine serum (FBS) Gibco ${ }^{\text {TM }}$ (Thermo Fischer Scientific) and $1 \%$ penicillin/streptomycin Gibco ${ }^{\mathrm{TM}}$ (Thermo Fischer Scientific) at $37^{\circ} \mathrm{C}$ in a humidified atmosphere containing $5 \% \mathrm{CO}_{2}$. Cells were transferred to a 50 $\mathrm{ml}$ tube and centrifuged at $750 \times \mathrm{g}$ for $5 \mathrm{~min}$ at $4{ }^{\circ} \mathrm{C}$. In a second time, cells were resuspended in RPMI with $10 \%$ FBS and centrifuged again to wash out any trace of antibiotics. Cells were seeded in a 96-well plate at $10^{5}$ cells per well in $100 \mu$ l of RPMI with $10 \%$ FBS. Plates were incubated for $20 \mathrm{~h}$ at $37^{\circ} \mathrm{C}$ with $5 \% \mathrm{CO}_{2}$. Cells of $\mathrm{C}$. violaceum following $16 \mathrm{~h}$ of culture with and without enzyme were diluted in RPMI with 10\% FBS and added to J774.1 cells; the different multiplicity of infection (MOI) of 1,10 , and 100 were tested and $\mathrm{MOI}$ of 1 was selected as $\mathrm{MOI}$ of 10 and 100 led to cytotoxic levels too high to be evaluated properly. In order to assess supernatant toxicity, $10 \mu \mathrm{l}$ of supernatant of the bacterial culture was added to $10^{5} \mathrm{~J} 774.1$ cells. After a centrifugation step $\left(10 \mathrm{~min}, 500 \times \mathrm{g}, 4^{\circ} \mathrm{C}\right)$ to bring bacteria in contact with macrophages, the plate was incubated for $1.5 \mathrm{~h}$ at $37^{\circ} \mathrm{C}$ with $5 \% \mathrm{CO}_{2}$. Supernatants were collected after centrifugation of $\left(5 \mathrm{~min}, 200 \times \mathrm{g}, 4^{\circ} \mathrm{C}\right)$. Cytotoxicity induced in $\mathrm{J} 774.1$ cells was quantitated by measuring the release of the cytosolic enzyme lactate dehydrogenase (LDH) in the culture medium using CyQUANT ${ }^{\mathrm{T}}$ LDH Cytotoxicity Assay Kit (Thermo Fischer Scientific) according to the manufacturer recommendations. Maximum LDH release was measured with the lysis buffer provided by the kit, added $45 \mathrm{~min}$ before the end of the infection. Negative control was measured by adding PBS to noninfected macrophage cells.

\section{Toxicity assay using planarians}

Freshwater planarians belonging to the Schmidtea mediterranea species (asexual clonal line CIW4) were used for the experiment. The planarians were maintained in autoclaved water at $19^{\circ} \mathrm{C}$ in the dark and fed twice a week with calf liver. The animals were starved for at least one week prior to the experiments. The water was changed every 2 days and did not contain antibiotics. Worms were manually selected to fall within a certain range of size, around $0.8-1 \mathrm{~cm}$ in length. Cells of $C$. violaceum from a 16-h culture with and without enzyme were washed twice in PBS and then resuspended at the desired concentrations in autoclaved water. In all, $4 \mathrm{ml}$ of the bacterial solution was transferred into a 12-well plate. Five planarians were added in each well and the plate was incubated at $19^{\circ} \mathrm{C}$ in the dark. Survival was monitored over 12 days. Experiments were conducted in duplicate. To follow the evolution of bacterial concentration in the wells, samples of tap water were taken from worm-containing wells and control wells without worms over time. Then, the samples were serially diluted and plated on an LB-Agar plate. Colony-forming units (CFUs) were counted after $48 \mathrm{~h}$ incubation at room temperature.

\section{Toxicity effect of $C$. violaceum supernatants against $S$. mediterranea}

To evaluate the bactericidal effect of $C$. violaceum supernatants against $S$. mediterranea planarian, supernatants from a 16-h culture in LB with and without enzyme of $C$. violaceum were collected by centrifugation and filtered at $0.22 \mu \mathrm{m}$. Freshwater planarians belonging to the Schmidtea mediterranea species (asexual clonal line CIW4) were used for the experiment. The planarians were maintained in autoclaved water at $19^{\circ} \mathrm{C}$ in the dark and fed twice a week with calf liver. The animals were starved for at least one week prior to the experiments. The water was changed every 2 days and did not contain antibiotics. Worms were manually selected to fall within a certain range of size, around $0.8-1 \mathrm{~cm}$ in length. Supernatants of $C$. violaceum from a $16 \mathrm{~h}$ culture with and without enzyme were resuspended at the desired concentrations in autoclaved water. In total, $4 \mathrm{ml}$ of the supernatant solution was transferred into a 12well plate. Five planarians were added in each well, and the plate was incubated at $19^{\circ} \mathrm{C}$ in the dark. Survival was monitored over 12 days. Experiments were conducted in duplicate.

\section{Reporting summary}

Further information on research design is available in the Nature Research Reporting Summary linked to this article.

\section{DATA AVAILABILITY}

All data used in this study, including raw data, are available upon request to the corresponding authors.

\section{CODE AVAILABILITY}

Codes used in this study are available upon request to the corresponding authors.

Received: 31 October 2020; Accepted: 25 March 2021; Published online: 22 April 2021

\section{REFERENCES}

1. Miller, M. B. \& Bassler, B. L. Quorum sensing in bacteria. Annu. Rev. Microbiol. 55, 165-199 (2001).

2. Déziel, E. et al. Analysis of Pseudomonas aeruginosa 4-hydroxy-2-alkylquinolines (HAQs) reveals a role for 4-hydroxy-2-heptylquinoline in cell-to-cell communication. Proc. Natl Acad. Sci. USA 101, 1339-1344 (2004).

3. Seyedsayamdost, M. R. et al. Quorum-sensing-regulated bactobolin production by Burkholderia thailandensis E264. Org. Lett. 12, 716-719 (2010).

4. McClean, K. H. et al. Quorum sensing and Chromobacterium violaceum: exploitation of violacein production and inhibition for the detection of $\mathrm{N}$ acylhomoserine lactones. Microbiology 143, 3703-3711 (1997).

5. Federle, M. J. \& Bassler, B. L. Interspecies communication in bacteria. J. Clin. Investig. 112, 1291-1299 (2003).

6. Evans, K. C. et al. Quorum-sensing control of antibiotic resistance stabilizes cooperation in Chromobacterium violaceum. ISME J. 12, 1263-1272 (2018).

7. Moura-Alves, P. et al. Host monitoring of quorum sensing during Pseudomonas aeruginosa infection. Science 366, eaaw1629 (2019).

8. Rémy, B. et al. Interference in bacterial quorum sensing: a biopharmaceutical perspective. Front. Pharmacol. 9, 203 (2018).

9. Guendouze, A. et al. Effect of quorum quenching lactonase in clinical isolates of Pseudomonas aeruginosa and comparison with quorum sensing inhibitors. Front. Microbiol. 8, 227 (2017).

10. Mahan, K. et al. Effects of signal disruption depends on the substrate preference of the lactonase. Front. Microbiol. 10, 3003 (2020).

11. Bergonzi, C. et al. Structural and biochemical characterization of AaL, a quorum quenching lactonase with unusual kinetic properties. Sci. Rep. 8, 11262 (2018).

12. Bergonzi, C., Schwab, M., Naik, T. \& Elias, M. The structural determinants accounting for the broad substrate specificity of the quorum quenching lactonase GcL. ChemBioChem 20, 1848-1855 (2019).

13. Koch, G. et al. Reducing virulence of the human pathogen Burkholderia by altering the substrate specificity of the quorum-quenching acylase PvdQ. Proc. Natl Acad. Sci. USA 111, 1568-1573 (2014).

14. Mion, S., Plener, L., Rémy, B., Daudé, D. \& Chabrière, É. Lactonase SsoPox modulates CRISPR-Cas expression in Gram-negative proteobacteria using AHL-based quorum sensing systems. Res. Microbiol. 170, 296-299 (2019).

15. Schwab, M. et al. Signal disruption leads to changes in bacterial community population. Front. Microbiol. 10, 611 (2019).

16. Huang, S., Bergonzi, C., Schwab, M., Elias, M. \& Hicks, R. E. Evaluation of biological and enzymatic quorum quencher coating additives to reduce biocorrosion of steel. PLOS ONE 14, e0217059 (2019).

17. Whiteley, M., Diggle, S. P. \& Greenberg, E. P. Progress in and promise of bacterial quorum sensing research. Nature 551, 313-320 (2017).

18. Hiblot, J., Gotthard, G., Elias, M. \& Chabriere, E. Differential active site loop conformations mediate promiscuous activities in the lactonase SsoPox. PLOS ONE 8 , e75272 (2013).

19. Morohoshi, T., Kato, M., Fukamachi, K., Kato, N. \& Ikeda, T. N-acylhomoserine lactone regulates violacein production in Chromobacterium violaceum type strain ATCC 12472. FEMS Microbiol. Lett. 279, 124-130 (2008).

20. Morohoshi, T., Fukamachi, K., Kato, M., Kato, N. \& Ikeda, T. Regulation of the violacein biosynthetic gene cluster by acylhomoserine lactone-mediated quorum sensing in Chromobacterium violaceum ATCC 12472. Biosci. Biotechnol. Biochem. 74, 2116-2119 (2010).

21. Stauff, D. L. \& Bassler, B. L. Quorum sensing in Chromobacterium violaceum: DNA recognition and gene regulation by the CviR receptor. J. Bacteriol. 193, 3871-3878 (2011).

22. Lima, D. C. et al. GeLC-MS-based proteomics of Chromobacterium violaceum: comparison of proteome changes elicited by hydrogen peroxide. Sci. Rep. 6, 28174 (2016). 
23. Wang, M. et al. Sharing and community curation of mass spectrometry data with global natural products social molecular networking. Nat. Biotech. 34, 828-837 (2016).

24. Carriot, N. et al. Integration of LC/MS-based molecular networking and classical phytochemical approach allows in-depth annotation of the metabolome of nonmodel organisms - the case study of the brown seaweed Taonia atomaria. Talanta 225, 121925 (2021)

25. Cheng, W.-J. et al. Quorum sensing inhibition and tobramycin acceleration in Chromobacterium violaceum by two natural cinnamic acid derivatives. Appl. Microbiol. Biotechnol. 104, 5025-5037 (2020).

26. Schuster, M., Joseph Sexton, D., Diggle, S. P. \& Peter Greenberg, E. Acylhomoserine lactone quorum sensing: from evolution to application. Annu. Rev. Microbiol. 67, 43-63 (2013).

27. Rasmussen, B. B. et al. Global and phylogenetic distribution of quorum sensing signals, Acyl homoserine lactones, in the family of vibrionaceae. Mar. Drugs 12, 5527-5546 (2014).

28. Liu, J. et al. "In-Group" communication in marine vibrio: a review of N-Acyl homoserine lactones-driven quorum sensing. Front. Cell. Infect. Microbiol. 8, 139 (2018).

29. Hong, K.-W., Koh, C.-L., Sam, C.-K., Yin, W.-F. \& Chan, K.-G. Complete genome sequence of Burkholderia sp. strain GG4, a betaproteobacterium that reduces 3Oxo-N-acylhomoserine lactones and produces different $\mathrm{N}$-acylhomoserine lactones. J. Bacteriol. 194, 6317-6317 (2012).

30. Girard, L. et al. Evidence of a large diversity of $\mathrm{N}$-acyl-homoserine lactones in symbiotic Vibrio fischeri strains associated with the Squid Euprymna scolopes. Microbes Environ. 34, 99-103 (2019).

31. Elias, M. et al. Structural basis for natural lactonase and promiscuous phosphotriesterase activities. J. Mol. Biol. 379, 1017-1028 (2008).

32. Hirano, S., Asamizu, S., Onaka, H., Shiro, Y. \& Nagano, S. Crystal structure of VioE, a key player in the construction of the molecular skeleton of violacein. J. Biol. Chem. 283, 6459-6466 (2008).

33. Hoshino, T. Violacein and related tryptophan metabolites produced by Chromobacterium violaceum: biosynthetic mechanism and pathway for construction of violacein core. Appl. Microbiol. Biotechnol. 91, 1463-1475 (2011).

34. Vinod, H. D. Canonical ridge and econometrics of joint production. J. Econom. 4, 147-166 (1976).

35. Leurgans, S. E., Moyeed, R. A. \& Silverman, B. W. Canonical correlation analysis when the data are curves. J. R. Stat. Soc. Ser. B Stat. Methodol. 55, 725-740 (1993).

36. González, I., Déjean, S., Martin, P. \& Baccini, A. CCA: an R package to extend canonical correlation analysis. J. Stat. Softw. 23, 1-14 (2008).

37. Carepo, M. S. P. et al. Identification of Chromobacterium violaceum genes with potential biotechnological application in environmental detoxification. Genet. Mol. Res. 3, 181-194 (2004).

38. Short, S. M., van Tol, S., MacLeod, H. J. \& Dimopoulos, G. Hydrogen cyanide produced by the soil bacterium Chromobacterium sp. Panama contributes to mortality in Anopheles gambiae mosquito larvae. Sci. Rep. 8, 8358 (2018).

39. Blumer, C. \& Haas, D. Mechanism, regulation, and ecological role of bacterial cyanide biosynthesis. Arch. Microbiol. 173, 170-177 (2000).

40. Sobin, B. A. \& Tanner, F. W. Anisomycin,1 a new anti-protozoan antibiotic. J. Am. Chem. Soc. 76, 4053-4053 (1954).

41. Zheng, X. et al. Biosynthesis of the pyrrolidine protein synthesis inhibitor anisomycin involves novel gene ensemble and cryptic biosynthetic steps. Proc. Natl Acad. Sci. USA 114, 4135-4140 (2017).

42. Hosoya, Y., Kameyama, T., Naganawa, H., Okami, Y. \& Takeuchi, T. Anisomycin and new congeners active against human tumor cell lines. J. Antibiot. 46, 1300-1302 (1993).

43. Rijavec, T. \& Lapanje, A. Hydrogen cyanide in the rhizosphere: not suppressing plant pathogens, but rather regulating availability of phosphate. Front. Microbiol. 7, 1785 (2016)

44. Choi, S. Y. et al. Chromobacterium violaceum delivers violacein, a hydrophobic antibiotic, to other microbes in membrane vesicles. Environ. Microbiol. 22, 705-713 (2020).

45. Aruldass, C. A., Masalamany, S. R. L., Venil, C. K. \& Ahmad, W. A. Antibacteria mode of action of violacein from Chromobacterium violaceum UTM5 against Staphylococcus aureus and methicillin-resistant Staphylococcus aureus (MRSA). Environ. Sci. Pollut. Res. 25, 5164-5180 (2018).

46. Sasidharan, A. et al. Antifungal activity of violacein purified from a novel strain of Chromobacterium sp. NIIST (MTCC 5522). J. Microbiol. 53, 694-701 (2015).

47. Laville, J. et al. Characterization of the hcnABC gene cluster encoding hydrogen cyanide synthase and anaerobic regulation by ANR in the strictly aerobic biocontrol agent Pseudomonas fluorescens CHAO. J. Bacteriol. 180, 3187-3196 (1998).

48. Van Dyke, N., Pickering, B. F. \& Van Dyke, M. W. Stm1p alters the ribosome association of eukaryotic elongation factor 3 and affects translation elongation. Nucleic Acids Res. 37, 6116-6125 (2009).
49. Batista, J. H. \& da Silva Neto, J. F. Chromobacterium violaceum pathogenicity: updates and insights from genome sequencing of novel Chromobacterium Species. Front. Microbiol. 8, 2213 (2017).

50. Durán, N. \& Menck, C. F. Chromobacterium violaceum: a review of pharmacological and industiral perspectives. Crit. Rev. Microbiol. 27, 201-222 (2001).

51. Chattopadhyay, M. K. Use of antibiotics as feed additives: a burning question Front. Microbiol. 5, 334 (2014).

52. Ghosh, R., Tiwary, B. K., Kumar, A. \& Chakraborty, R. Guava leaf extract inhibits quorum-sensing and Chromobacterium violaceum induced lysis of human hepatoma cells: whole transcriptome analysis reveals differential gene expression. PLoS ONE 9, e107703 (2014).

53. Winzer, K. et al. The Pseudomonas aeruginosa lectins PA-IL and PA-IIL are controlled by quorum sensing and by RpoS. J. Bacteriol. 182, 6401-6411 (2000).

54. Zinger-Yosovich, K., Sudakevitz, D., Imberty, A., Garber, N. C. \& Gilboa-Garber, N. Production and properties of the native Chromobacterium violaceum fucosebinding lectin (CV-IIL) compared to homologous lectins of Pseudomonas aeruginosa (PA-IIL) and Ralstonia solanacearum (RS-IIL). Microbiology 152, 457-463 (2006).

55. Kumar, A. S., Bryan, J. N. \& Kumar, S. R. Bacterial quorum sensing molecule N-3oxo-dodecanoyl-L-homoserine lactone causes direct cytotoxicity and reduced cell motility in human pancreatic carcinoma cells. PLOS ONE 9, e106480 (2014).

56. Hagstrom, D., Cochet-Escartin, O., Zhang, S., Khuu, C. \& Collins, E.-M. S. Freshwater planarians as an alternative animal model for neurotoxicology. Toxicol. Sci. 147, 270-285 (2015).

57. Poirier, L. et al. Enzymatic degradation of organophosphorus insecticides decreases toxicity in planarians and enhances survival. Sci. Rep. 7, 15194 (2017).

58. Poirier, L., Ghigo, É., Daudé, D. \& Chabrière, É. Modèles alternatifs (6) - La planaire, un modèle animal original pour la toxicologie. Med. Sci. 35, 544-548 (2019).

59. Poirier, L., Plener, L., Daudé, D. \& Chabrière, E. Enzymatic decontamination of paraoxon-ethyl limits long-term effects in planarians. Sci. Rep. 10, 3843 (2020).

60. Abnave, $\mathrm{P}$. et al. Screening in planarians identifies MORN2 as a key component in LC3-associated phagocytosis and resistance to bacterial infection. Cell Host Microbe 16, 338-350 (2014).

61. Ballestriero, F. et al. Antinematode activity of violacein and the role of the insulin/ IGF-1 pathway in controlling violacein sensitivity in Caenorhabditis elegans. PLoS ONE 9, e109201 (2014).

62. Swem, L. R. et al. A quorum-sensing antagonist targets both membrane-bound and cytoplasmic receptors and controls bacterial pathogenicity. Mol. Cell $\mathbf{3 5}$ 143-153 (2009).

63. Wenemoser, D., Lapan, S. W., Wilkinson, A. W., Bell, G. W. \& Reddien, P. W. A molecular wound response program associated with regeneration initiation in planarians. Genes Dev. 26, 988-1002 (2012).

64. Hiblot, J., Gotthard, G., Chabriere, E. \& Elias, M. Characterisation of the organophosphate hydrolase catalytic activity of Sso Pox. Sci. Rep. 2, 779 (2012).

65. Hraiech, S. et al. Inhaled lactonase reduces Pseudomonas aeruginosa quorum sensing and mortality in rat pneumonia. PLOS ONE 9, e107125 (2014).

66. Favre, L. et al. Metabolome and proteome changes between biofilm and planktonic phenotypes of the marine bacterium Pseudoalteromonas lipolytica TC8. Biofouling 34, 132-148 (2018).

67. Pluskal, T., Castillo, S., Villar-Briones, A. \& Orešič, M. MZmine 2: modular framework for processing, visualizing, and analyzing mass spectrometry-based molecular profile data. BMC Bioinform. 11, 395 (2010)

68. Chong, J. et al. MetaboAnalyst 4.0: towards more transparent and integrative metabolomics analysis. Nucleic Acids Res. 46, W486-W494 (2018)

69. Nothias, L.-F. et al. Environmentally friendly procedure based on supercritical fluid chromatography and tandem mass spectrometry molecular networking for the discovery of potent antiviral compounds from Euphorbia semiperfoliata. J. Nat. Prod. 80, 2620-2629 (2017).

70. Tautenhahn, R. et al. An accelerated workflow for untargeted metabolomics using the METLIN database. Nat. Biotechnol. 30, 826-828 (2012).

71. Dührkop, K. et al. SIRIUS 4: a rapid tool for turning tandem mass spectra into metabolite structure information. Nat. Methods 16, 299-302 (2019).

72. Bergonzi, C., Schwab, M. \& Elias, M. The quorum-quenching lactonase from Geobacillus caldoxylosilyticus: purification, characterization, crystallization and crystallographic analysis. Acta Cryst. F. 72, 681-686 (2016).

73. Stepanović, S., Vuković, D., Dakić, I., Savić, B. \& Švabić-Vlahović, M. A modified microtiter-plate test for quantification of staphylococcal biofilm formation. J. Microbiol. Methods 40, 175-179 (2000).

74. Chessa, J.-P., Petrescu, I., Bentahir, M., Van Beeumen, J. \& Gerday, C. Purification, physico-chemical characterization and sequence of a heat labile alkaline metalloprotease isolated from a psychrophilic Pseudomonas species. Biochimica et. Biophysica Acta (BBA) - Protein Struct. Mol. Enzymol. 1479, 265-274 (2000).

75. Castillo-Juárez, I., García-Contreras, R., Velázquez-Guadarrama, N., Soto-Hernández, M. \& Martínez-Vázquez, M. Amphypterygium adstringens anacardic acid 
mixture inhibits quorum sensing-controlled virulence factors of Chromobacterium violaceum and Pseudomonas aeruginosa. Arch. Med. Res. 44, 488-494 (2013).

76. von Rohr, M. R., Furrer, G. \& Brandl, H. Effect of iron and phosphate on bacterial cyanide formation determined by methemoglobin in two-dimensional gradient microcultivations. J. Microbiol. Methods 79, 71-75 (2009).

\section{ACKNOWLEDGEMENTS}

This work was financially supported by the French Ministry of Higher Education, Research and Innovation, the University of Toulon (Ph.D. grant of N. Carriot), the Direction Générale de l'Armement (DGA) (Ph.D. grant of S. Mion), and Investissements d'avenir program (Méditerranée Infection 10-IAHU-03) of the French Agence Nationale de la Recherche (ANR). This work also received support from the ANR Astrid research program (RESSAC) from the DGA. The authors wish to thank Dr B. Paix (Toulon University, MAPIEM) for the helpful discussions concerning multi-omics analyses, Dr S. Greff (Aix-Marseille University, IMBE) for the acquisition of LC-MS data, Prof. J-L. Mège for providing macrophages, and P. Decloquement and S. Azza for the acquisition of proteomic data. LC-MS experiments were conducted on the regional platform MALLABAR funded by the Institute of Ecology and Environment (INEE) of the French National Centre for Scientific Research (CNRS), the Total Foundation and the French Sud-PACA regional council.

\section{AUTHOR CONTRIBUTIONS}

S.M., L.P., A.O.M., E.C., G.C., and D.D. conceived the study. S.M., N.C., L.P., A.O.M., G.C., and D.D. designed the experimental studies. S.M., N.C., J.L., and L.P. performed the experiments. All authors have a substantial contributions to the paper, were involved in the writing, approved the final version, and are accountable for all aspects of the work.

\section{COMPETING INTERESTS}

E.C. has a patent WO2014167140 A1 licensed to Gene\&GreenTK. S.M., L.P., E.C., and D.D. have filled the patent FR3093894. S.M., L.P., D.D., and E.C. report personal fees from Gene\&GreenTK during the conduct of the study. The remaining authors declare that the research was conducted in the absence of any commercial or financial relationships that could be construed as a potential conflict of interest.

\section{ADDITIONAL INFORMATION}

Supplementary information The online version contains supplementary material available at https://doi.org/10.1038/s41522-021-00211-w.

Correspondence and requests for materials should be addressed to E.C., G.C. or D.D.

Reprints and permission information is available at http://www.nature.com/ reprints

Publisher's note Springer Nature remains neutral with regard to jurisdictional claims in published maps and institutional affiliations.

(i) Open Access This article is licensed under a Creative Commons Attribution 4.0 International License, which permits use, sharing, adaptation, distribution and reproduction in any medium or format, as long as you give appropriate credit to the original author(s) and the source, provide a link to the Creative Commons license, and indicate if changes were made. The images or other third party material in this article are included in the article's Creative Commons license, unless indicated otherwise in a credit line to the material. If material is not included in the article's Creative Commons license and your intended use is not permitted by statutory regulation or exceeds the permitted use, you will need to obtain permission directly from the copyright holder. To view a copy of this license, visit http://creativecommons. org/licenses/by/4.0/.

(c) The Author(s) 2021 